\title{
Sequential Tolerance Control in Discrete Parts Manufacturing
}

Tom M. Cavalier(tmc7@psu.edu, 814-863-2371)

E. Amine Lehtihet (1vo@psu.edu, 814-863-2350)

Industrial \& Manufacturing Engineering

207 Hammond Building

The Pennsylvania State University

University Park, PA 16802

\begin{abstract}
The machining of complex parts typically involves a sequence of $n$ operations on $m$ machine tools. Conventional tolerance control specifies a fixed set point for each such operation and permissible variation about this set point to insure compliance with tolerance specifications. However, this approach may be inadequate for complex, low-volume, highvalue added parts. This paper introduces the concept of Sequential Tolerance Control (STC), an approach that uses real-time measurement information at the completion of a stage to exploit available space inside a dynamic feasible zone and reposition the set point for subsequent operations so as to optimize the production of an acceptable part. It is also demonstrated that the measurement information acquired during STC can be used to compensate for systematic variation such as tool wear. STC is then used in the context of an implicit enumeration approach to select an optimal subset of technological processes required to execute a process plan. Finally, a probabilistic approach to the problem of optimal selection of technological processes under conventional tolerance control is presented and the First Order Second Moment Method (FOSMM) is used to estimate yield.
\end{abstract}

Introduction: Interchangeability in mechanical assemblies and satisfactory functional aptitude of parts and products are only possible when component dimensions and other geometric features are produced inside prescribed tolerance zones. The drawing or print of a part translates the above requirement into a sketch of exact part geometry augmented by dimensional and geometric tolerance information; tolerance information specifies permissible deviations from perfect geometry. In machining, final specifications of a mechanical part are arrived at via a logical, chronological sequence of material removal operations. This sequence may involve several machine tools with each machine tool performing one or more material removal operations, under one or more distinct set-ups. During the processing of a complex part, design specification datums and manufacturing operations datums do not always coincide. This leads to the commonly encountered situation where, even under the best of manufacturing plans, a large subset of part specifications are satisfied by the combination of two or more manufactured dimensions. Knowing that the output of each manufacturing operation (dimension produced) $x_{j}$ will fall within a certain tolerance range such that $x_{j} \in\left[x_{j \min }, x_{j \max }\right]$ leads to the classical stack up of tolerances for those specifications produced by the combination of two or more manufacturing operations. If $c_{i} \in\left[c_{i \min }, c_{i \max }\right], i=1, \ldots, n$ is the set of design constraints (dimensions) and $S_{i}$ the subset of manufacturing operations which combine to produce constraint $c_{i}$, the stack up problem can be formulated as follows. 
$\operatorname{minimize} \sum_{i=1}^{n} z_{i}$

subject to

$$
\begin{array}{ll}
\sum_{j \in S_{i}} t_{j}+z_{i}=b_{i} & i=1, \ldots, n \\
t_{j} \geq L P C_{j} & \mathrm{j}=1, \ldots, m
\end{array}
$$

where

$$
\begin{aligned}
& z_{i}=\text { residual tolerance in constraint } c_{i} \\
& t_{j}=\left(x_{j \max }-x_{j \min }\right)=\text { tolerance of operation } x_{j} \\
& b_{i i}=\left(c_{i \max }-c_{i \min }\right)=\text { tolerance of constraint } c_{i} \\
& L P C_{j}=\text { lower tolerance for capability (precision) of operation } x_{j}
\end{aligned}
$$

The problem of selecting the precision required of technological processes under a conventional tolerance control strategy is commonly known as the tolerance chart allocation problem. The problem has been treated in a number of publications ([1] - [16]). It remains the main tool used by process planners in industry to determine permissible variation, i.e., for the output $x_{j}$ of each manufacturing operation in the process plan so that design specifications $c_{i}$ are satisfied. The longevity of this tool is proof of its usefulness in the mass production of relatively low cost parts where a small percentage of defects can be tolerated. An example of this production strategy is depicted in Figure 1(a). Variability limits around a fixed set point are determined for each manufacturing operation prior to the production run. At the completion of operation $j$, one of two outcomes is possible:

(a) If $x_{j} \in\left[x_{j \min }, x_{j \max }\right]$, the part proceeds to operation $j+1$

(b) If $x_{j} \notin\left[x_{j \min }, x_{j \max }\right]$, the part is diverted from the normal flow with the possibility of reentering the flow of operations if it is economically salvageable.

The important characteristic of this strategy is that machine tool settings for operation $j+1$ are the same for all parts which have satisfied operation $j$.

While this strategy is appropriate for the mass, batch or automated transfer line production of relatively low cycle time, low value-added workpieces, it presents serious problems for the machining of complex, low volume, high value-added parts. Typical workpieces include aircraft structural frames, aircraft engine components and housings, large combat vehicle parts, or high precision precious metal parts in jewelry or medical applications. A helicopter gear housing or gear, or a self-propelled gun turret part require large numbers of machining operations executed over several days. The primary objective in these instances is to maximize the quality and chance of producing a good part. A scrapped part towards the end of the manufacturing plan represents a large economic loss and delay. Conventional tolerance chart analysis in this case tries to satisfy the objective by specifying overly conservative bounds on the variability of each manufacturing operation relative to the initial fixed set point. Conservative bounds on variability, in turn, require more accuracy and precision from manufacturing equipment and greater control over every environmental or 
machine tool/tool/fixture related component of the error budget. This invariably leads to more financial expenditures, higher manufacturing costs and higher penalties in case of defects.

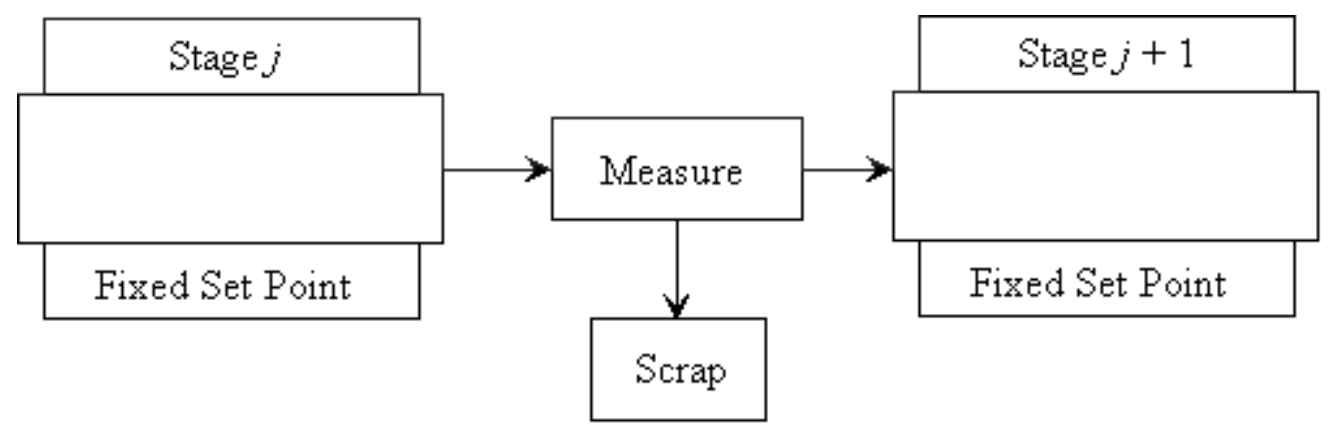

(a)

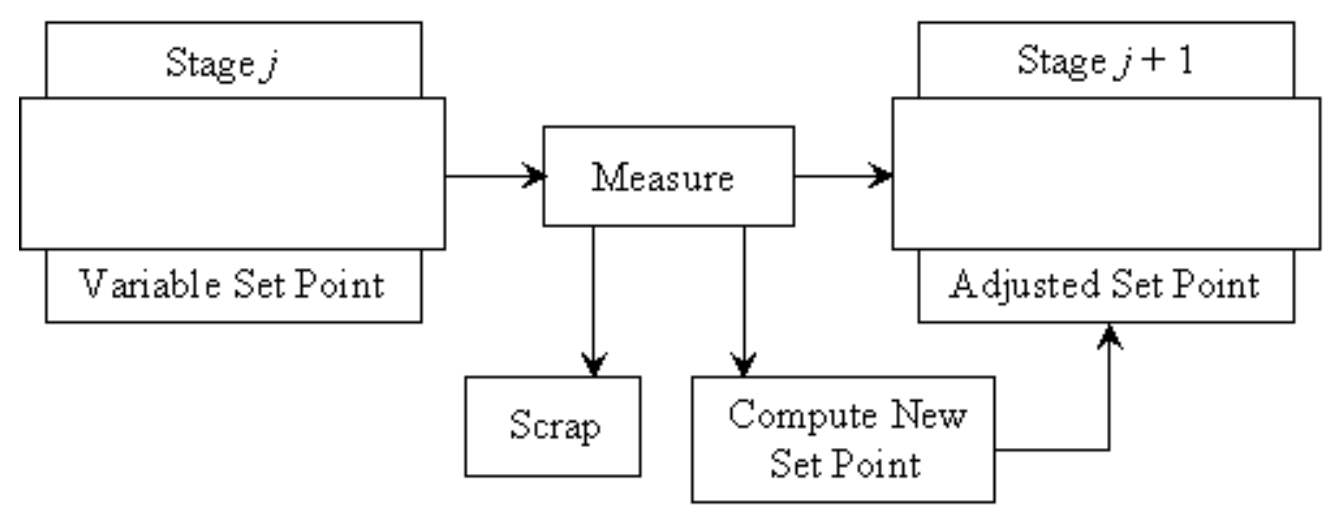

(b)

Figure 1. (a) Conventional Tolerance Control, (b) Sequential Tolerance Control

This paper outlines the development and technological implementation of an alternative approach to conventional tolerance control in these cases. It is based on the concept of Sequential Tolerance Control (STC) as the part moves through a sequence of $n$ operations prescribed in the process plan. Its objective is to sequentially and selectively adjust the target point of machining operations to maximize final output quality.

This paper begins by using a simple example to illustrate the concept of STC. We then provide the details of the general methodology, the implementation for a specific test part, and some simulation results. Next, a methodology of STC with tool-wear correction is provided. Several tool-wear compensation strategies are then examined, and a practical implementation on a part and simulation results are presented.

This is followed by an implicit enumeration procedure that incorporates the idea of STC. 
This procedure is designed to solve the tolerance allocation problem by selecting a set of processes that results in an acceptable yield rate at minimum cost. The discussion includes numerical examples illustrating the implicit enumeration process. The implicit enumeration framework is also applicable when using conventional tolerance control, and the results obtained from STC are contrasted with those from conventional tolerance control.

Finally, an implicit enumeration procedure using conventional tolerance control and the First Order Second Moment Method (FOSMM) for system yield computation is presented. The procedure solves the tolerance allocation problem by selecting a set of processes that satisfy a yield constraint and minimize cost. The procedure is then applied to two tolerance chart allocation problems and yield computations using FOSMM are validated using Monte Carlo simulation.

Sequential Tolerance Control - Concept and Example: STC in machining was introduced by Fraticelli et al [17] while a related approach was studied by Duret [18]. The development presented here is due to Fraticelli et al [17]. It is predicated on the ability to obtain accurate measurements after one or more manufacturing operations have been carried out, and act to conserve or modify the set points or target values of the next operation(s) in order to maximize the probability of producing a good part. With the wide availability of contact coordinate measuring machines (CMMs), optical CMMs, machine-tool-mounted touchtrigger probes and other precision measurement equipment, the ability to acquire measurements on or off the shop floor has grown tremendously. For complex, high valueadded workpieces, frequent pauses for measurements at intermediate steps of the process plan are already a reality and do not constitute an additional constraint. What is proposed then is to make far better use of these measurements to effect the outcome of subsequent operation(s) and maximize the chance of success. The concept of adaptive control of tolerances over the set of machining operations prescribed in the process plan is illustrated in Figure 1(b). The important characteristic of this approach is that after a measurement is acquired at the end of manufacturing operation $j$, an examination of the resulting feasible region for manufacturing operations $j+1, j+2, \ldots, n$ is carried out. A new set point is then computed for operations $j+$ $1, j+2, \ldots, n$ so as to maximize the chance of success. The new set point is passed on to operation $j+1$ which is then executed; measurement of the output of $j+1$ is acquired and the process repeated until all operations are exhausted and manufacturing is complete. A simple example will illustrate the mechanics of this approach.

Figure 2 shows a simple hypothetical part with three design constraints, $c_{1}=1.0 \pm 0.001, c_{2}=0.75 \pm 0.002, \mathrm{c}_{3}=0.5 \pm 0.002$. Manufacturing operations $X_{1}, X_{2}$, and $X_{3}$ are executed sequentially to satisfy these constraints. Each operation is shown as a vector between two parallel faces; the origin of the vector represents the surface used for location and the extremity of the vector indicates the surface generated by the operation. Note that the manufacturing plan is not very realistic and is used strictly to illustrate the concept. The relationship between dimensions for $x_{1}, x_{2}, x_{3}$ produced by the operations and the constraints $c_{1}, c_{2}$, and $c_{3}$ are given by the following system of equations: 


$$
\left(\begin{array}{l}
0.999 \\
0.748 \\
0.498
\end{array}\right) \leq\left(\begin{array}{ccc}
1 & 0 & -1 \\
-1 & 1 & 1 \\
1 & -1 & 0
\end{array}\right)\left(\begin{array}{l}
x_{1} \\
x_{2} \\
x_{3}
\end{array}\right) \leq\left(\begin{array}{l}
1.001 \\
0.752 \\
0.502
\end{array}\right)
$$

The conventional approach to tolerance control in this case would analyze the system and fix the variability of each manufactured dimension around a target point. An obvious target point here is the triplet $\left(x_{1}, x_{2}, x_{3}\right)^{t}=(2.250,1.750,1.250)$ and a possible allocation of the stack up of tolerances is $\left(\delta_{1}, \delta_{2}, \delta_{3}\right)^{t}=( \pm 0.0005, \pm 0.001, \pm 0.0005)$ where $\delta_{i}$ is the tolerance range of each operation. Upon execution, control of the dimensions $x_{1}, x_{2}, x_{3}$ within these prescribed bounds guarantees $100 \%$ good parts. If the output of an operation falls outside its bounds, it is withdrawn from the flow.

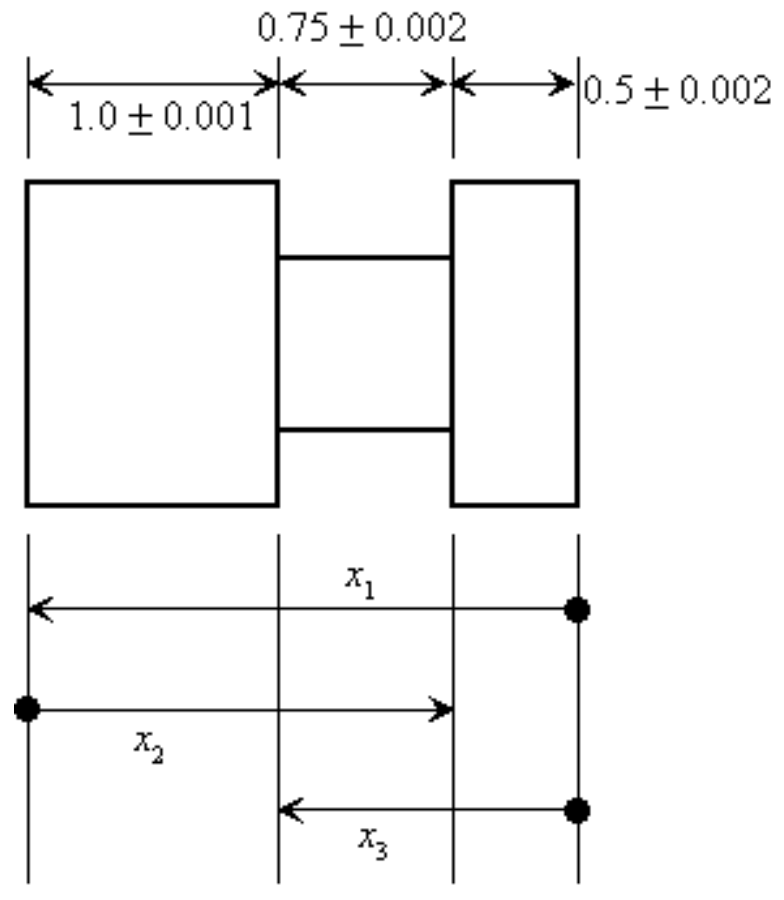

Figure 2. A Simple Hypothetical Part

The proposed STC scheme would start with the same set point, but uses the result of operation $j$ to find a new set point for operation $j+1$. Figures 3-6 show several instances of the resulting feasible zone (plotted in a coordinate system where the origin is the first set point $\left.(2.250,1.750,1.250)^{t}\right)$ after execution of operation $x_{1}$.

Figure 3 assumes a realization for $x_{1}=2.2505$ and shows the resulting feasible domain of operations $x_{2}$ and $x_{3}$ plotted relative to initial set point $\mathbf{o}_{1}$. If the set point $\mathbf{o}_{1}$ is kept for the next operations, as would be the case under conventional tolerance control, operation $x_{2}$ appears safe, but a deviation for operation $x_{3}$ in excess of 0.0005 in the negative direction would produce a bad part. STC would then probably move the new set point to point $\mathbf{0}_{2}$ for 
operations $x_{2}$ and $x_{3}$.

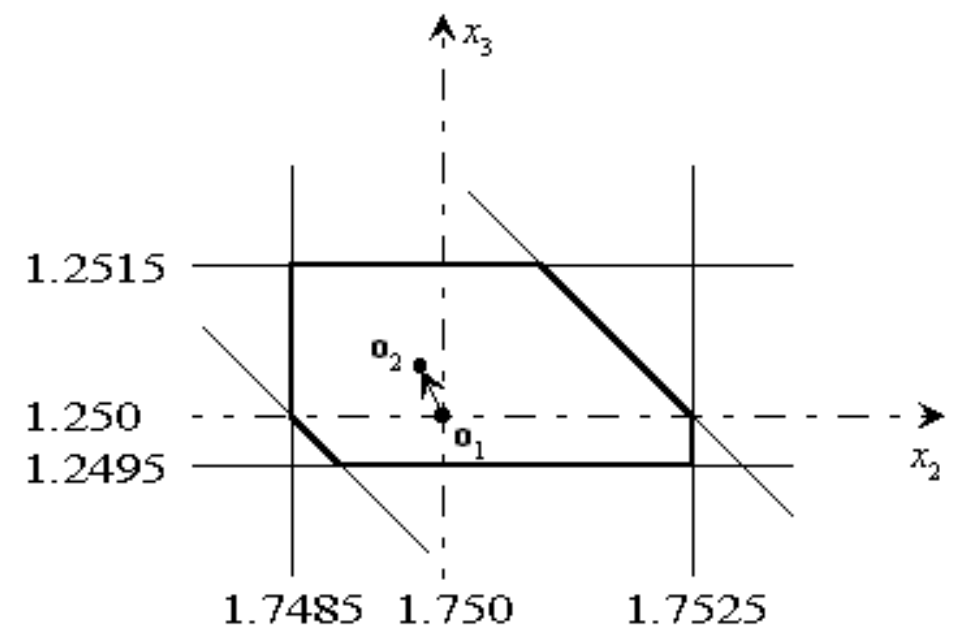

Figure 3. Resulting Feasible Domain when $x_{1}=2.2505$

Figure 4 assumes a realization for $x_{1}=2.2490$; any realization of $x_{3}$ greater than 1.250 (the original set point for $x_{3}$ ) under conventional tolerance control would produce a bad part. STC control would reset the new set point to point $\mathbf{o}_{2}$ for operations $x_{2}$ and $x_{3}$.

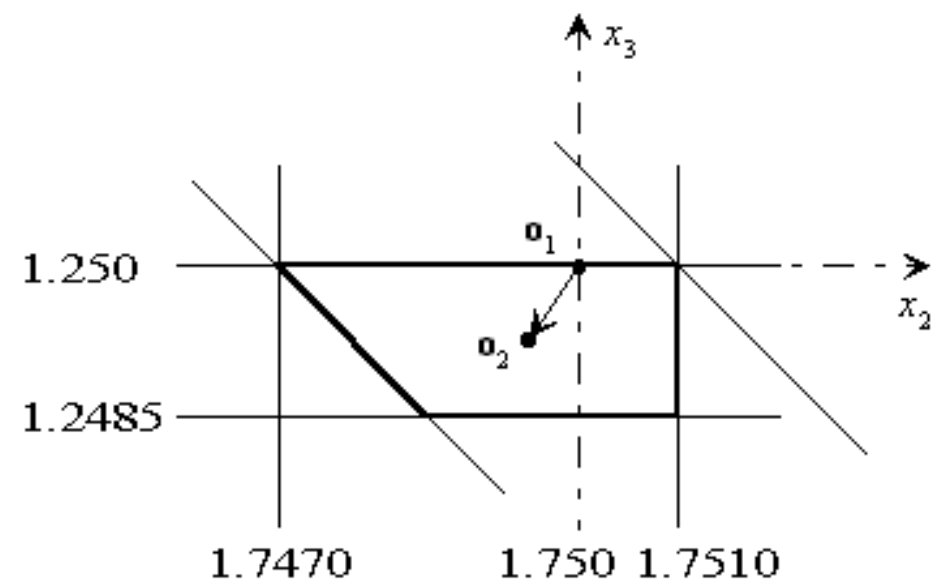

Figure 4. Resulting Feasible Domain when $x_{1}=2.2490$

Figure 5 assumes a realization for $x_{1}=2.2515$; any realization of $x_{2}$ less than 1.7495 or $x_{3}$ less than 1.2505 would produce bad parts if set point $\mathbf{o}_{1}$ is kept for operations $x_{2}$ and $x_{3}$. STC would move the set point to $\mathbf{0}_{2}$. 


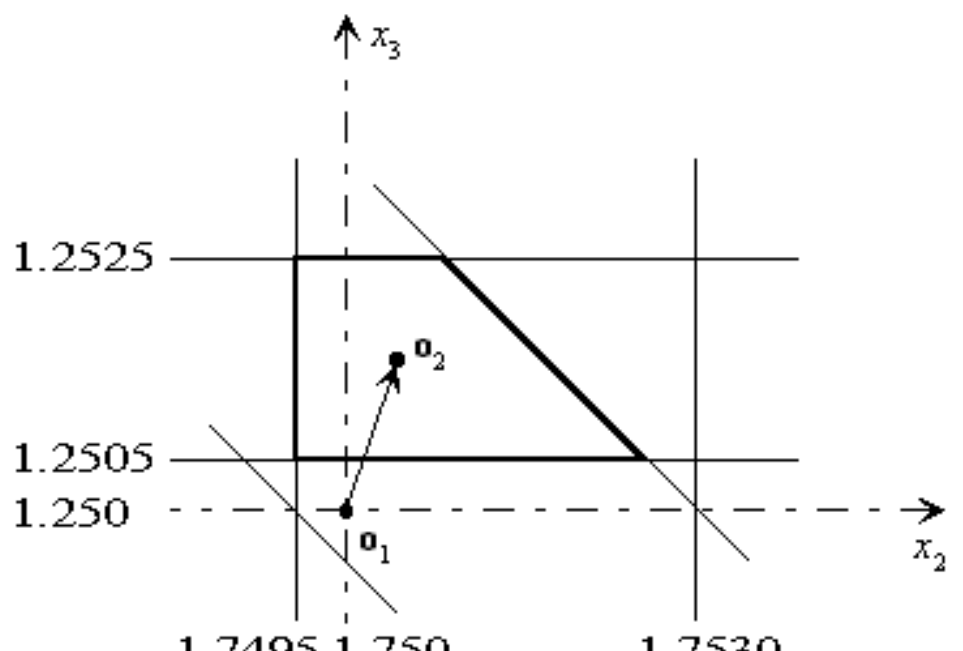

$1.74951 .750 \quad 1.7530$

Figure 5. Resulting Feasible Domain when $x_{1}=2.2515$

Finally, Figure 6 assumes a realization of $x_{1}=2.2520$; it is clear that any realization of $x_{2}$ less than 1.750 or $x_{3}$ less than 1.251 would miss the feasible region if set point $\mathbf{0}_{1}$ is kept. STC would reset the new target to point $\mathbf{o}_{2}$ for operations $x_{2}$ and $x_{3}$.

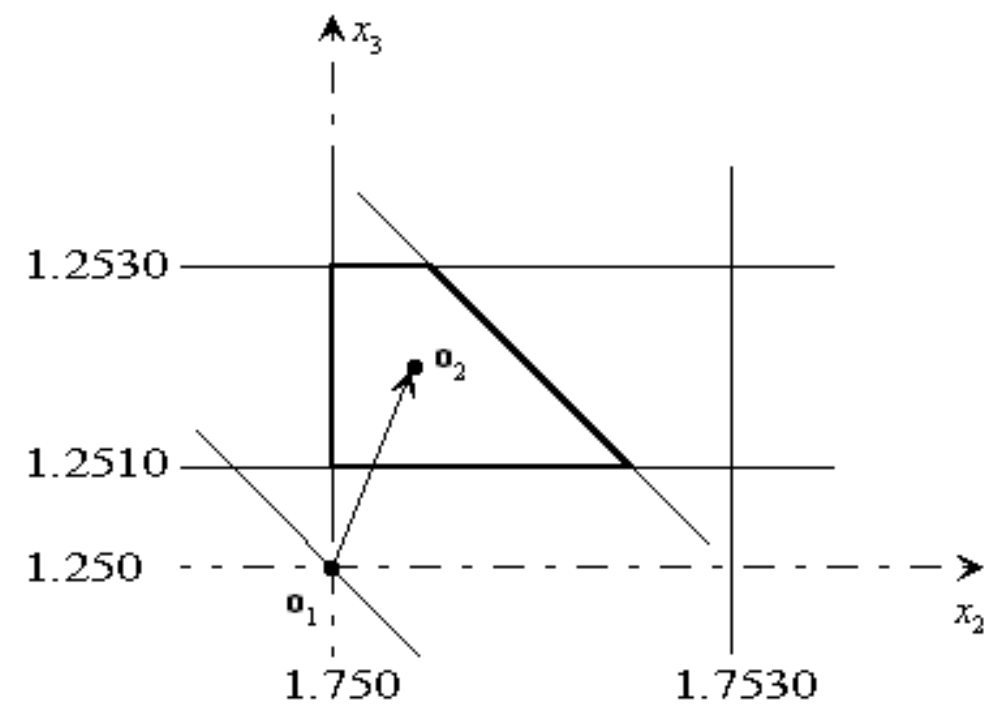

Figure 6. Resulting Feasible Domain when $x_{1}=2.2520$

Sequential Tolerance Control - Generalization and Solution: This section generalizes the adjustment procedure introduced in the previous section to address the process plan of a complex part. A complex part can be thought of as incorporating $n$ design and process planning constraints $c_{i} \in\left[c_{i \min }, c_{i \max }\right], i=1, \ldots, n$ along a particular direction, satisfied by the selective combination of $n$ manufacturing operations producing manufactured dimensions. In general, the solution domain will be represented by the set of linear inequalities, 
$\mathbf{c}_{\min } \leq \mathbf{A x} \leq \mathbf{c}_{\max }$, or written in terms of the constraint rows,

$$
c_{i \min } \leq \mathbf{a}_{i} \mathbf{x} \leq c_{i \max } \quad i=1, \ldots, n
$$

where

$$
\begin{aligned}
& \mathbf{a}_{i}=\left(a_{i 1}, a_{i 2}, \ldots, a_{i 3}\right)=\text { the } i \text { th row of the matrix } \mathbf{A} \\
& \mathbf{x}=\left(x_{1}, x_{2}, \ldots, x_{n}\right) \text { and } x_{j}=\text { manufactured dimension produced by operation } X_{j}
\end{aligned}
$$

The above formulation represents a feasible region enclosed by a convex polyhedral set. Since each constraint is bounded by an upper and lower bound, each constraint gives rise to a pair of parallel hyperplanes separated by a distance equal to the tolerance range of the constraint. In addition, the vast majority of coefficients $a_{i j}$ will be such that $a_{i j}=0, \pm 1$; thus, the feasible region is some sort of "skewed" hypercube delineated by hyperplanes that are parallel to or at 45 degrees to the coordinate axes. Prior to machining, the target point of all machining operations is set at the center of this original feasible region. This point represents the location inside the feasible region that will allow the greatest degree of variability of planned machining operations. After the first operation $X_{1}$ is executed, the dimension produced by this operation is inspected and its value $x_{1}=\hat{x}_{1}$ can be substituted into constraint set (2). This is equivalent to slicing the original feasible region by a plane orthogonal to direction $X_{1}$ at the realized value. If the intersection is not empty, it will represent the new feasible region for the $n-1$ remaining operations. As illustrated in the previous examples, the original set point is not necessarily the best location for the $n-1$ remaining operations. The task of finding a new target point that maximizes the chance of success is the first problem to be solved. One possible approach is to fit the largest possible $(n-1)$-dimensional sphere inside the new feasible region. By fitting the largest sphere into the feasible region, each subsequent machining operation is guaranteed a tolerance range at least as wide as the diameter of the sphere.

Toward this end, suppose that we want to find the largest sphere that can be inscribed in a polyhedral set defined by $\mathbf{A x} \leq \mathbf{c}$. Let the $i$ th constraint of this system be $\mathbf{a}_{i} \mathbf{x} \leq c_{i}$ and note that this constraint forms part of the boundary of the feasible region as in Figure 7 . The point on the boundary of the inscribed circle that is closest to the $i$ th constraint can be defined in terms of the gradient of the constraint $\mathbf{a}_{i}$, the circle center $\mathbf{y}=\left(y_{1}, \ldots, y_{n}\right)^{t}$, and the radius $r$, as $\mathbf{y}+r \mathbf{a}_{i}^{t}\left\|\mathbf{a}_{i}\right\|$ where $\left\|\mathbf{a}_{i}\right\|=\left(\sum_{j=1}^{n} a_{i j}^{2}\right)^{1 / 2}$. Note that if this point satisfies the defining constraint, that is $\mathbf{a}_{i}\left(\mathbf{y}+r \mathbf{a}_{i}^{t} /\left\|\mathbf{a}_{i}\right\|\right)=\mathbf{a}_{i} \mathbf{y}+r\left\|\mathbf{a}_{i}\right\|^{2} /\left\|\mathbf{a}_{i}\right\|=\mathbf{a}_{i} \mathbf{y}+r\left\|\mathbf{a}_{i}\right\| \leq c_{i}$, then all points within the circle must also satisfy this constraint. 


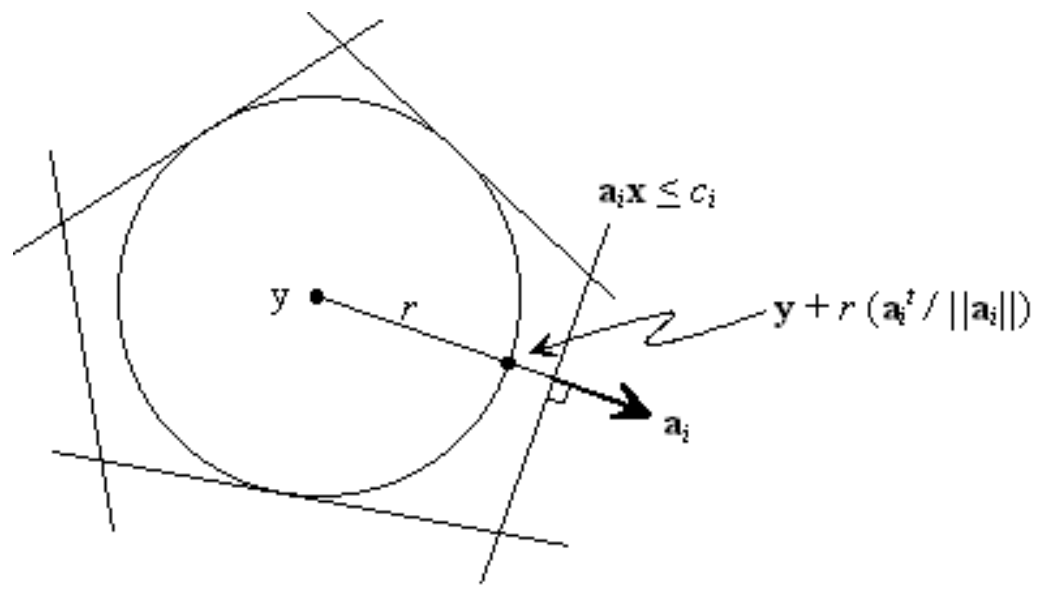

Figure 7. Inscribing the Largest Sphere

Thus, the problem of determining the largest circle which can be enclosed in the constraint set $\{\mathbf{A} \mathbf{x} \leq \mathbf{c}\}=\left\{\mathbf{a}_{i} \mathbf{x} \leq c_{i}, i=1, \ldots, m\right\}$, can be written as

maximize $r$

subject to

$$
\begin{aligned}
& \mathbf{a}_{i} \mathbf{y}+r\left\|\mathbf{a}_{i}\right\| \leq c_{i} \quad i=1, \ldots, m \\
& \mathbf{y}=\left(y_{1}, \ldots, y_{n}\right)^{t} \quad \text { unrestricted } \\
& r \geq 0
\end{aligned}
$$

Note that the constraint $\mathbf{a}_{i} \mathbf{y}+r\left\|\mathbf{a}_{i}\right\| \leq c_{i}$ can also be written in the form

$$
\sum_{j=1}^{n} a_{i j} y_{j}+r\left(\sum_{j=1}^{n} a_{i j}^{2}\right)^{\frac{1}{2}} \leq c_{i} \text {. }
$$

Let $r_{\max }$ be the optimal objective value associated with this problem. In cases where the sphere of maximum radius occurs at a unique location, the target value of the next machining operation will be associated with the center of the sphere. However, in many feasible regions, the largest sphere can be fitted into one of many possible locations. This indicates that the maximum allocation of future tolerances can be achieved by several different target values. In these cases, it may be desirable to aim for the midpoint of the extreme target values. This maximizes the probability of producing an actual cut that falls within the region of maximum tolerances.

To determine the extreme target values for a particular coordinate direction, say $x_{j}$, we simply minimize and maximize the corresponding component $y_{j}$ of the center while maintaining $r=$ $r_{\max }$ in formulation (3) (See Figure 8). This can be done in a straightforward manner by solving two linear programming problems: 
minimize $y_{j}$ and maximize $y_{j}$

subject to

$$
\begin{aligned}
& \mathbf{a}_{i} \mathbf{y} \leq c_{i}-r_{\max }\left\|\mathbf{a}_{i}\right\| \quad i=1, \ldots, m \\
& \mathbf{y}=\left(y_{1}, \ldots, y_{n}\right)^{t} \text { unrestricted }
\end{aligned}
$$

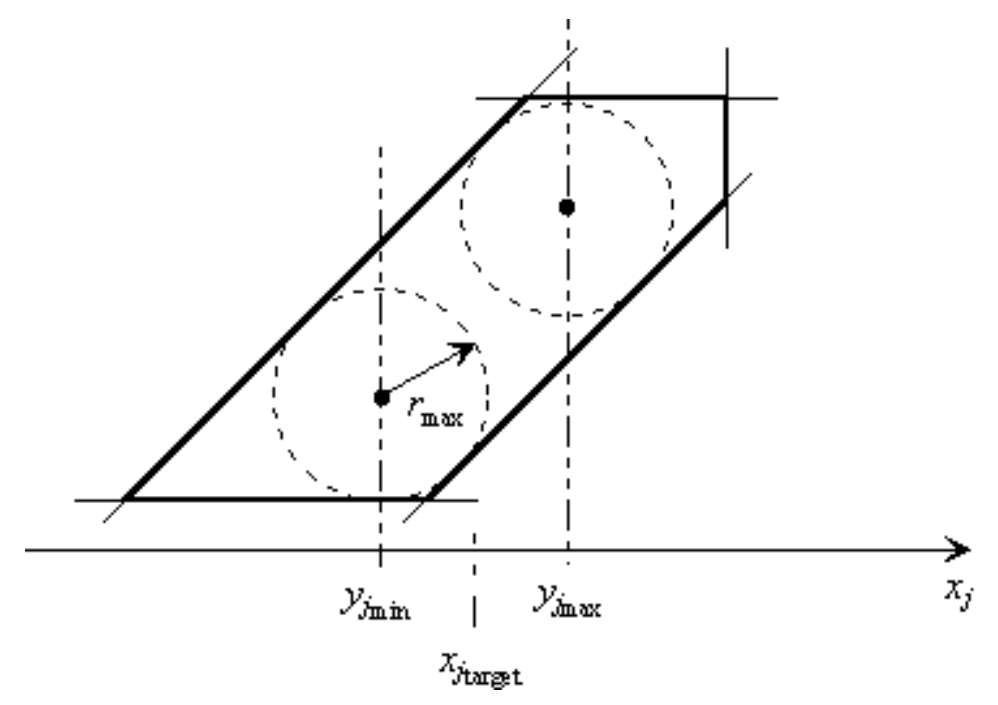

Figure 8. Finding the Extreme Positions of the Sphere

Let us now summarize the proposed adaptive method of sequentially calculating target values for $n$ successive machining operations. The process begins by initially setting operation $X_{1}$ at its nominal value. After the first cut is made, the variable corresponding to the first machining operation is set equal to the value that was actually produced, that is $x_{1}=\hat{x}_{1}$.

Now assume that $k-1$ operations have been performed and the actual values are $x_{i}=\hat{x}_{i}, i=1, \ldots, k-1$. Then the target value of the $k$ th operation is found as follows.

First, find the radius of the largest sphere that can be inscribed in the feasible region, which has now been reduce to $n-k+1$ dimensions. This is done by solving the linear programming problem:

maximize $r$

subject to

$$
\begin{aligned}
& \sum_{j=k}^{n} a_{i j} y_{j}-r\left(\sum_{j=k}^{n} a_{i j}^{2}\right)^{\frac{1}{2}} \geq c_{i \min }-\sum_{j=1}^{k-1} a_{i j} \hat{x}_{j} \quad i=1, \ldots, n \\
& \sum_{j=k}^{n} a_{i j} y_{j}+r\left(\sum_{j=k}^{n} a_{i j}^{2}\right)^{\frac{1}{2}} \leq c_{i \max }-\sum_{j=1}^{k-1} a_{i j} \hat{x}_{j} \quad i=1, \ldots, n \\
& \mathbf{y}=\left(y_{k}, \ldots, y_{n}\right)^{t} \text { unrestricted } \\
& r \geq 0
\end{aligned}
$$


Denoting by $r_{\max }$ the optimal objective value of the foregoing linear program, the extreme values associated with operation $X_{k}$ are found by solving

minimize $y_{k}$ and maximize $y_{k}$

subject to

$$
\begin{aligned}
& \sum_{j=k}^{n} a_{i j} y_{j} \geq c_{i \min }-\sum_{j=1}^{k-1} a_{i j} \hat{x}_{j}+r_{\max }\left(\sum_{j=k}^{n} a_{i j}^{2}\right)^{\frac{1}{2}} \quad i=1, \ldots, n \\
& \sum_{j=k}^{n} a_{i j} y_{j} \leq c_{i \max }-\sum_{j=1}^{k-1} a_{i j} \hat{x}_{j}-r_{\max }\left(\sum_{j=k}^{n} a_{i j}^{2}\right)^{\frac{1}{2}} \quad i=1, \ldots, n \\
& \mathbf{y}=\left(y_{k}, \ldots, y_{n}\right)^{t} \text { unrestricted }
\end{aligned}
$$

If the optimal solutions to these two linear programs are denoted $y_{k \min }$ and $y_{k \max }$, respectively, then the target value of operation $X_{k}$ is $\left(y_{k \min }+y_{k \max }\right) / 2$. Based on this setting for operation $X_{k}$, an actual value is realized and the process is repeated. This sequential adjustment of target values is continued until all operations have been performed.

Sequential Tolerance Control - Implementation: The previously described strategy was coded in FORTRAN and embedded in a larger simulation module designed to run conventional and sequential tolerance control approaches side by side. The manufacturing operations prescribed in the process plan are executed in the chronological order given by the plan. To execute a given manufacturing operation, the simulation module starts by generating a random deviate from a given density function. The random deviate is applied to the fixed target of the conventional tolerance control procedure to simulate variability of the executed operation; the same random deviate is applied to the adjusted set point of the sequential strategy for the same purpose. For each processed part, and for each tolerance control procedure, the simulation module keeps track of all constraints which are violated for a direct evaluation of the ability of STC to exploit available space inside a dynamic feasible zone and maximize the chance of success.

A cast steel drive hub (Eary and Johnson [2]) was chosen as a test part. This part and its process plan are shown in Figure 9 and Table 1, respectively. The tolerance control procedures are applied to the seven (7) axial design constraints shown on the drawing (five of these constraints link the six parallel surfaces of the part; two additional constraints result from the hardening requirement of $0.015 \pm 0.010$ at each end). This part presents several interesting characteristics for tolerance control. It involves three distinct machining processes (turning, milling, and grinding) with six distinct set-ups or datum surfaces. While machine tools create real surfaces, the heat treating operation produces an imaginary surface representing the depth of the hardened case. These operations combine in such a manner that five out of the seven axial design constraints are satisfied by subsets of two or three operations and their corresponding tolerance stack up. Incoming raw material shape has an overall length of $2.000 \pm 0.010$ and the process plan calls for removal of 0.030 at each end by facing operations, leaving 0.010 at each end again for removal by grinding operations. Other 
manufacturing operations cut directly into the stock.

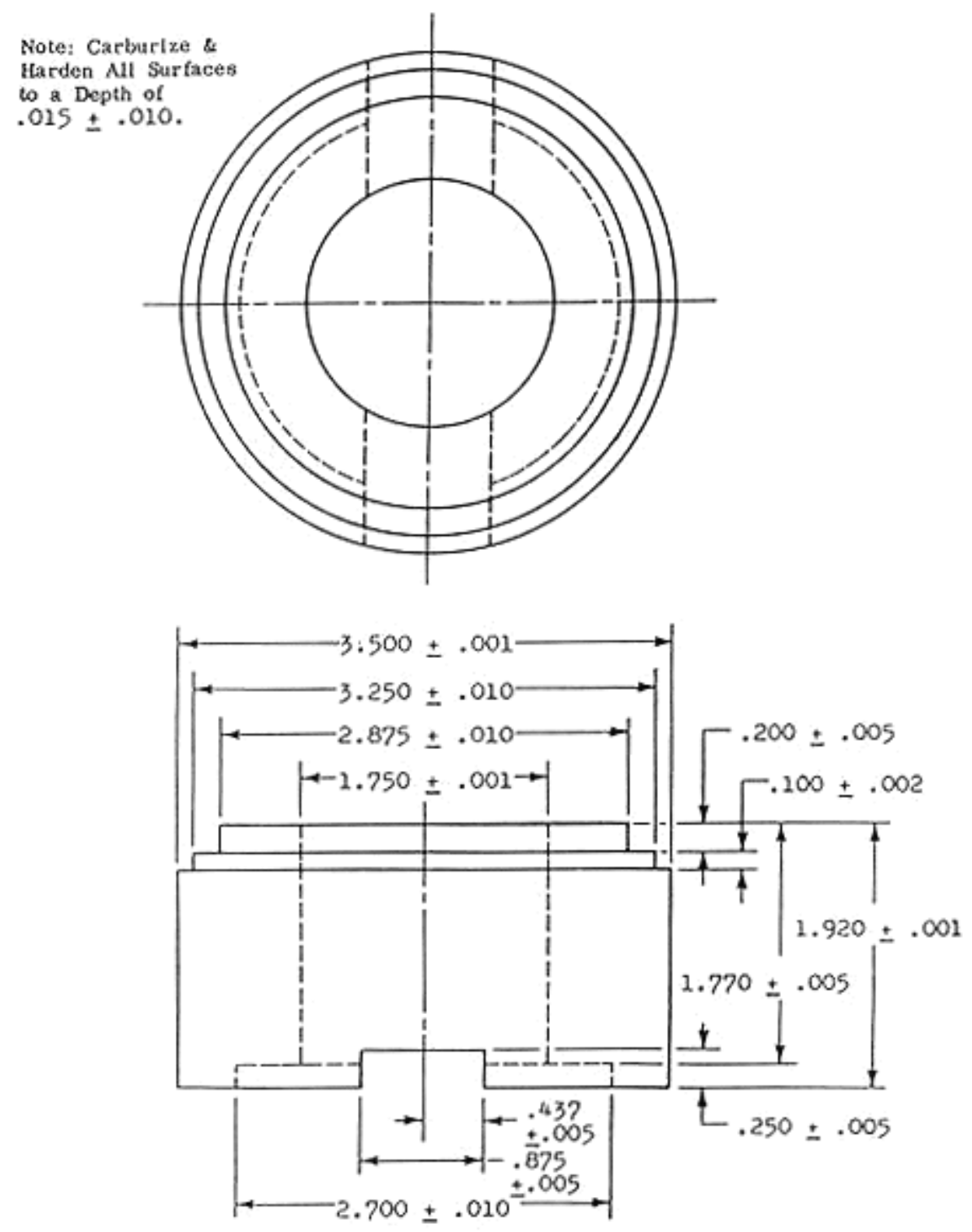

Figure 9. Cast Steel Drive Hub (Eary and Johnson [2])

Table 1. Process Plan (Eary and Johnson [2])

\begin{tabular}{|c|l|l|}
\hline $\begin{array}{l}\text { Operation } \\
\text { Number }\end{array}$ & Machine or Process & Operation Performed \\
\hline 5 & Bench & $\begin{array}{l}\text { Receiving inspection } \\
\text { Chuck on O.D.; Face one surface; Core drill \& } \\
\text { ream center hole to dimension. }\end{array}$ \\
\hline
\end{tabular}




\begin{tabular}{|c|c|c|}
\hline 20 & Turret Lathe & $\begin{array}{l}\text { Chuck on expanding stub mandrel; Locate on } \\
\text { machined face; Turn O.D., face, counterbore, and } \\
\text { remove burrs. }\end{array}$ \\
\hline 30 & Turret Lathe & $\begin{array}{l}\text { Chuck on expanding mandrel; Locate on second } \\
\text { face; Form steps and remove burrs. }\end{array}$ \\
\hline 40 & Simplex Mill & $\begin{array}{l}\text { Locate on surface opposite stepped surface; Mill } \\
\text { slot. }\end{array}$ \\
\hline 50 & Carb. \& H.T. Equip & Pack carburize and harden. \\
\hline 60 & Rotary Surface Grinder & Locate on slotted face. Grind opposite surface. \\
\hline 70 & Rotary Surface Grinder & Locate on ground surface. Grind opposite surface. \\
\hline 80 & Internal Grinder & Chuck on O.D.; Grind I.D. \\
\hline 90 & External Cyl. Grinder & Chuck on expanding mandrel; Grind O.D. \\
\hline 100 & Bench & Final Inspection. \\
\hline
\end{tabular}

Figure 10 shows a convenient graphical representation of constraints and manufacturing operations used to satisfy these constraints. The system of equations controlling compliance with the constraints is easily derived by inspection of the constraint and manufacturing graphs. The constraint graph shows explicit process planning and design constraints between pairs of parallel surfaces for easy visualization of objectives to be satisfied. The manufacturing graph shows the chronological execution of manufacturing operations $x_{i}, i=1, \ldots, 10$, where each operation $x_{i}$ is represented by a vector. The origin of the vector represents the datum or locating surface and the tip of the vector points to the surface generated by the tool. As an example, the subset of operations combining to satisfy constraint $c_{3-4}$ which controls hardened depth on the left hand side are determined as follows: first, locate surfaces 3 and 4 on the manufacturing graph; next find the shortest path, riding manufacturing operation vectors, which relates surface 3 to surface 4 ; by inspection, this path goes right along $x_{9}$ to node 11 , then left along $x_{2}$ to node 2 and finally right along $x_{7}$ to node 4 ; keeping track of the directions traveled allows the pair of planes generated by constraint $c_{3-}$ ${ }_{4}$ to be written as $c_{3-4 \min } \leq x_{9}-x_{2}+x_{7} \leq c_{3-4 \max }$. 


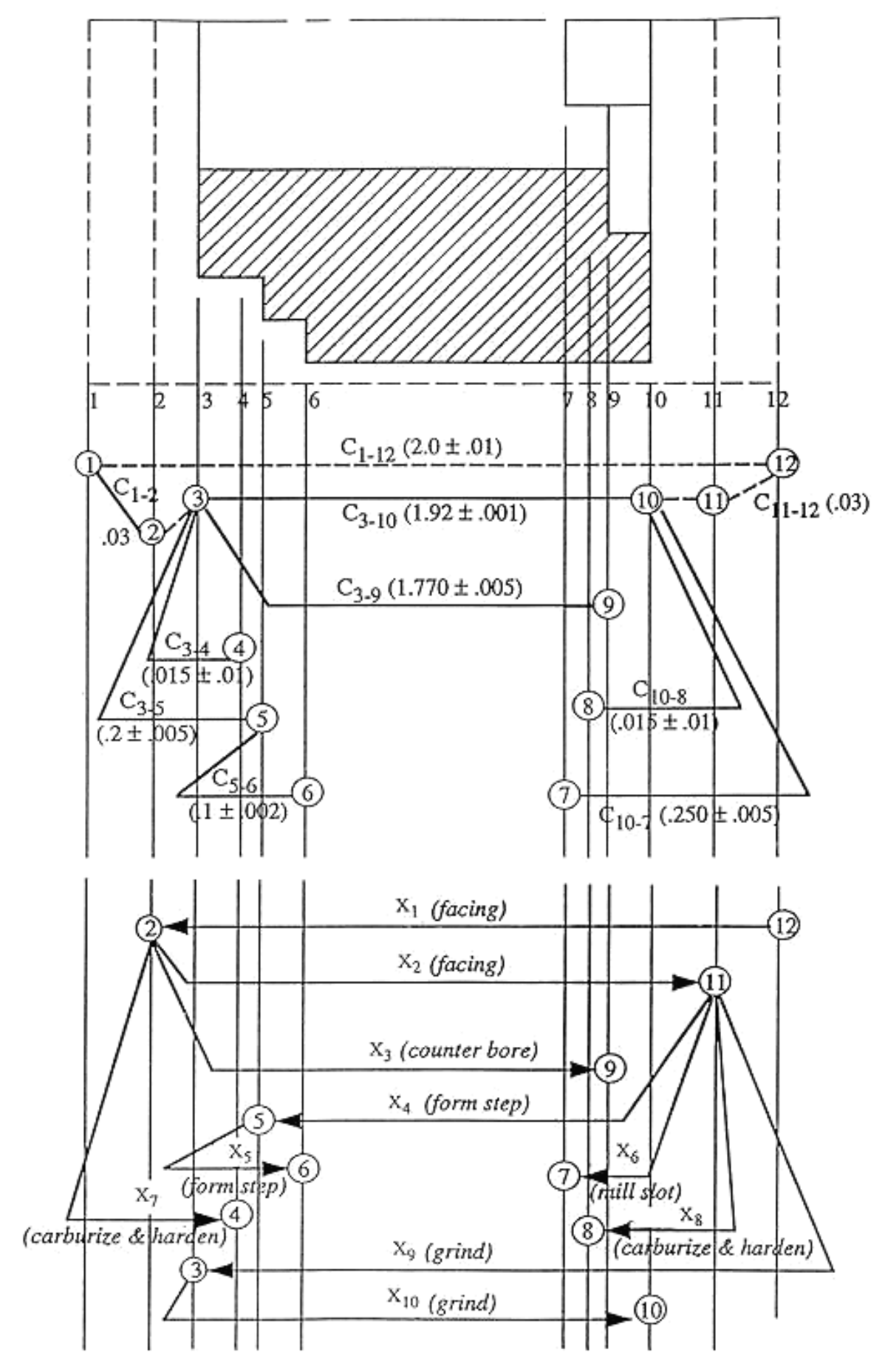

Figure 10. Graphical Representation of Constraints

The complete formulation of this problem is given below. The first set of constraints represents design constraints explicit on the part print. The second set of constraints refers to process planning constraints imposed by manufacturing to insure proper execution of the 
facing and grinding operations taken at each end of the workpiece. They will control the variability of the depth of cut $c_{d i-j}$ of the facing and grinding operations and insure that all depths of cut are neither too deep nor too shallow. Variable $L$ in this formulation refers to the overall length of the incoming raw material obtained by forging. Note that the heat treating operation generates $x_{7}$ and $x_{8}$ simultaneously and with the same variability; thus $x_{7}=x_{8}$.

Design constraints:

$$
\begin{aligned}
& c_{3-10 \min } \leq x_{10} \leq c_{3-10 \max } \\
& c_{3-9 \min } \leq x_{9}-x_{2}+x_{3} \leq c_{3-9 \max } \\
& c_{3-4 \min } \leq x_{9}-x_{2}+x_{7} \leq c_{3-4 \max } \\
& c_{8-10 \min } \leq x_{8}-x_{9}+x_{10} \leq c_{8-10 \max } \\
& c_{3-5 \min } \leq x_{9}-x_{4} \leq c_{3-5 \max } \\
& c_{5-6 \min } \leq x_{5} \leq c_{5-6 \max } \\
& c_{7-10 \min } \leq x_{6}-x_{9}+x_{10} \leq c_{7-10 \max }
\end{aligned}
$$

Process planning constraints:

$$
\begin{aligned}
& c_{d 1-2 \min } \leq L-x_{1} \leq c_{d 1-2 \max } \\
& c_{d 2-3 \min } \leq x_{2}-x_{9} \leq c_{d 2-3 \max } \\
& c_{d 10-11 \min } \leq x_{9}-x_{10} \leq c_{d 10-11 \max } \\
& c_{d 11-12 \min } \leq x_{1}-x_{2} \leq c_{d 11-12 \max }
\end{aligned}
$$

The comparative evaluation of the two strategies by way of simulation was implemented as follows:

(a) The initial simulation run was carried out using the original set point solution to the conventional tolerance control problem. The reader will easily verify that set point $x_{i}, i=1, \ldots, 10$ and permissible variability $\delta x_{i}, i=1, \ldots, 10$ given by the vector below constitute a feasible (and optimal in this case) solution to the conventional tolerance control problem. 


$$
\left(\begin{array}{c}
x_{1} \pm \delta x_{1} \\
x_{2} \pm \delta x_{2} \\
x_{3} \pm \delta x_{3} \\
x_{4} \pm \delta x_{4} \\
x_{5} \pm \delta x_{5} \\
x_{6} \pm \delta x_{6} \\
x_{7} \pm \delta x_{7} \\
x_{8} \pm \delta x_{8} \\
x_{9} \pm \delta x_{9} \\
x_{10} \pm \delta x_{10}
\end{array}\right)\left(\begin{array}{l}
1.970 \pm 0.002 \\
1.940 \pm 0.002 \\
1.780 \pm 0.002 \\
1.730 \pm 0.004 \\
0.100 \pm 0.002 \\
0.260 \pm 0.003 \\
0.025 \pm 0.007 \\
0.025 \pm 0.007 \\
1.930 \pm 0.001 \\
1.920 \pm 0.001
\end{array}\right)
$$

With the target points for each operation given by the values above, and variability of each executed operation confined to permissible variation $\pm \delta x_{i}$ given above, both control strategies should produce no defective parts and the sequential control strategy need not make any set point adjustments. This initial run at the above conditions is then a good check on the validity of the simulation code. Random deviates for this and subsequent simulation runs were sampled from uniform distributions with range equal to $\pm \delta x_{i}$. This choice is appropriate in the absence of factual information regarding the distribution of each $x_{i}$. Each run simulated the production of 50 parts.

(b) Subsequent simulation runs were designed to test the ability of STC to exploit available space inside the feasible zone and optimize production of good parts. This ability was evaluated by systematically increasing the ranges $\pm \delta x_{i}$ by $10 \%, 20 \%, 30 \%, 40 \%$ and $50 \%$ above initial values in (a) which guarantee $0 \%$ defects. These increments would force conventional tolerance control to produce more and more defects and test the ability of STC to absorb and compensate for this increased lack of precision. Note that constraints $c_{3-10}$ and $c_{5-6}$ are satisfied directly by operations $x_{10}$ and $x_{5}$, respectively, with no stack up. So $x_{10}$ and $x_{5}$ were not candidates for increased variability in the test since they are already using their full tolerance range and STC would have no means of compensating for additional increase in the ranges of these two operations.

Sequential Tolerance Control - Simulation Results: Figure 11 shows a plot of simulation results. The graph shows the number of defects under conventional and sequential control as the tolerance ranges of seven of the ten operations are incrementally made worse. The graph clearly shows the ability of STC to absorb some of the deterioration in precision of the process. As the variability range of operations increases by $30 \%$ above initial conventional values, STC performs five times better than conventional control; when the range of permissible variability deteriorates to $50 \%$ above initial values, sequential control still manages to perform three times better than conventional control. Where STC could not compensate, the amount by which the specification was violated was invariably smaller than the corresponding conventional control case. This is due to the dynamic set point centering at the basis of the procedure. Note also that sampling from uniform distributions constitutes, 
in effect, worst case conditions for the evaluation. The number of defects generated by both methods would be expected to be less had we sampled from distributions with decreasing probabilities for extreme values at the tails.

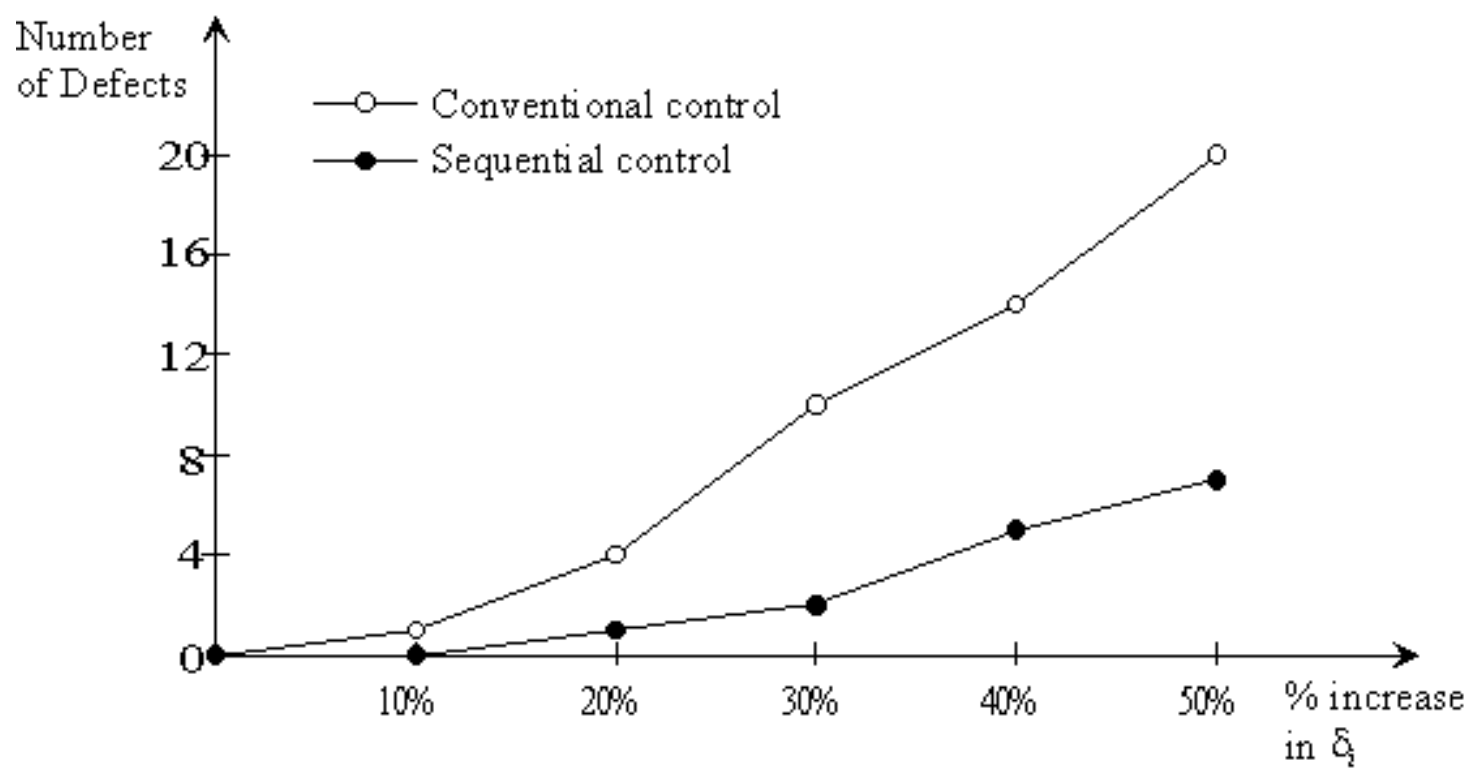

Figure 11. Simulation Results

The previous evaluation solved the "mechanics" problem of the procedure and clearly demonstrated its potential. It proceeded on the basis of measurements and set point adjustments after each chronological operation of the process plan. In general, measurement and set point adjustment after each distinct operation in the process plan would probably not be necessary and run the risk of being too disruptive of the manufacturing sequence. Any process plan must take advantage of associations of operations when they are compatible with a single set up, machine tool kinematics and tool holding capacity. Some associations under the same set-up are imposed by the nature and tightness of tolerances, and any break in the set-up would seriously compromise the ability to satisfy these tolerances due to fixturing uncertainty. Every process plan, however, has "natural" breaks in the sequence when a new datum system is called for or when the part moves to the next machine tool or process. These natural breaks in the process are certainly good candidates for evaluation of the resultant feasible zone and new set point adjustment. They must, however, be considered together with the magnitude of the tolerance zone for each constraint, the coefficients of each constraint, the chronological order in which constraints are satisfied, the degree to which the constraint set can be decomposed into disjoint blocks as well as the relative cost of each additional measurement break and set point adjustment.

Tool-Wear Compensation Using STC: Random variation, however, is not the only disturbance that affects a process. Cutting tools do wear with time and the gradual loss of cutting tool material introduces a systematic error component in the output of a machining operation. This systematic variation is often assumed to be a linear function of time. (See, for example, [19]-[26]). Our own experiments (See Figures 12 and 13), with simple facing and 
plunge cuts on steel workpieces using a steel cutting grade of carbide, clearly show a linear temporal drift of the manufactured dimensions. It is thus assumed that variation caused by non-repeatable (random) errors and tool wear can be adequately modeled by a momentary density function with constant variance and a mean that drifts linearly with time (or number of workpieces).

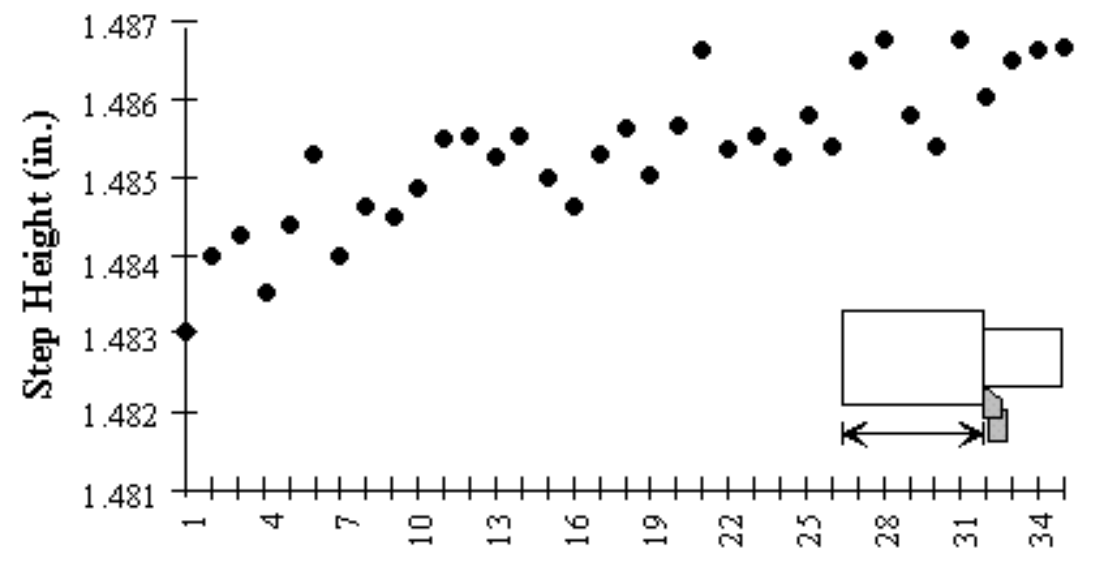

\section{Part Number}

Figure 12. Drift due to Tool Wear: Facing Cuts

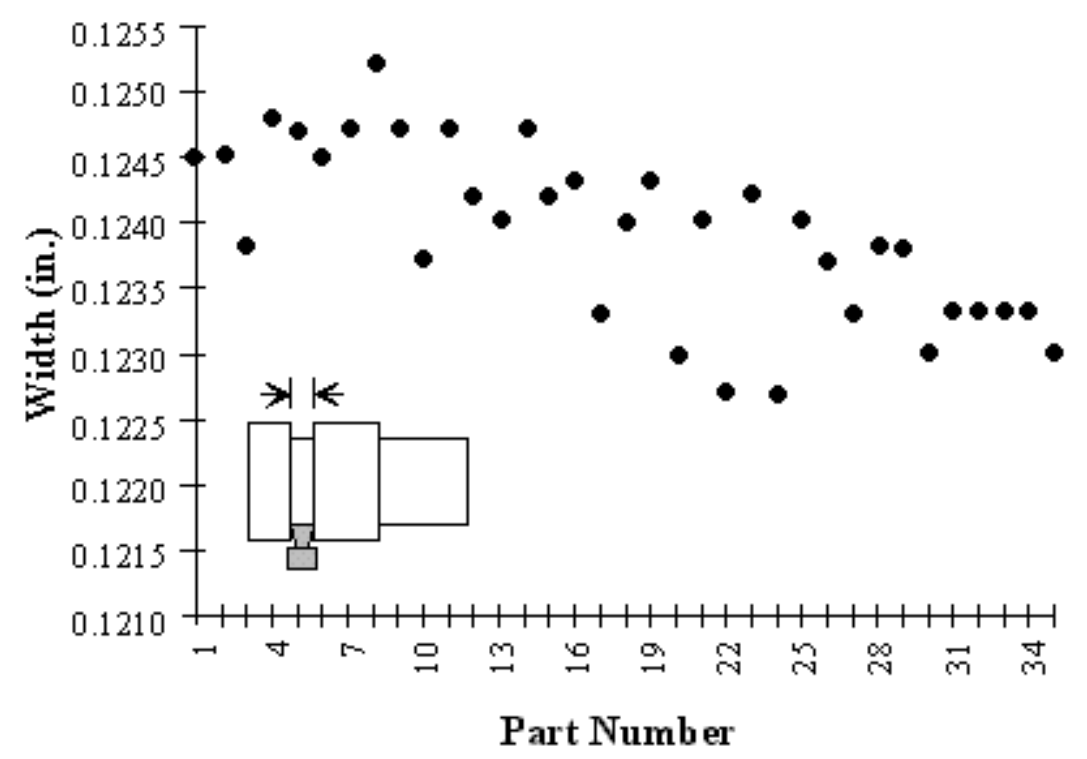

Figure 13. Drift due to Tool Wear: Plunge Cuts

Since STC is predicated on the ability to acquire information on the output of prior operations to adjust the set point of subsequent operations, Fraticelli et al [27] proposed using the acquired information to compensate for both random and systematic (tool wear) variation where appropriate. Compensation for drift due to tool wear would require no additional 
measurements or adjustments, and the additional computational time would be insignificant. The development presented here is based on Fraticelli et al [27].

The concept of STC with tool-wear correction is illustrated in Figure 14. The basic procedure of deriving a set point based upon actual process outputs is the STC procedure previously outlined. That is, given that operation $j$ has been completed and measured, the adjusted set point, $\mathbf{o}_{2}$, for operation $j+1$ is computed using formulations (4) and (5). At this point, however, the deviations for operation $j+1$ in the previously produced parts are examined for a systematic trend. If it is inferred that a trend exists, a correction factor, equal to the projected deviation for operation $j+1$ in the current part, is calculated. A corrected set point, $\mathbf{o}_{2}{ }^{\prime}$, which reflects the anticipated effect of tool wear, is obtained by subtracting the correction factor from $\mathbf{o}_{2}$. In summary, set point $\mathbf{o}_{2}$ remains the most desirable point in the feasible region, but the effect of tool wear will likely move the realized value away from this point. In order to move the realized value to location $\mathbf{0}_{2}$, set point $\mathbf{0}_{2}{ }^{\prime}$ is the new target. If the effect of tool wear is close to its anticipated value, the mean process output will occur near $\mathbf{0}_{2}$.

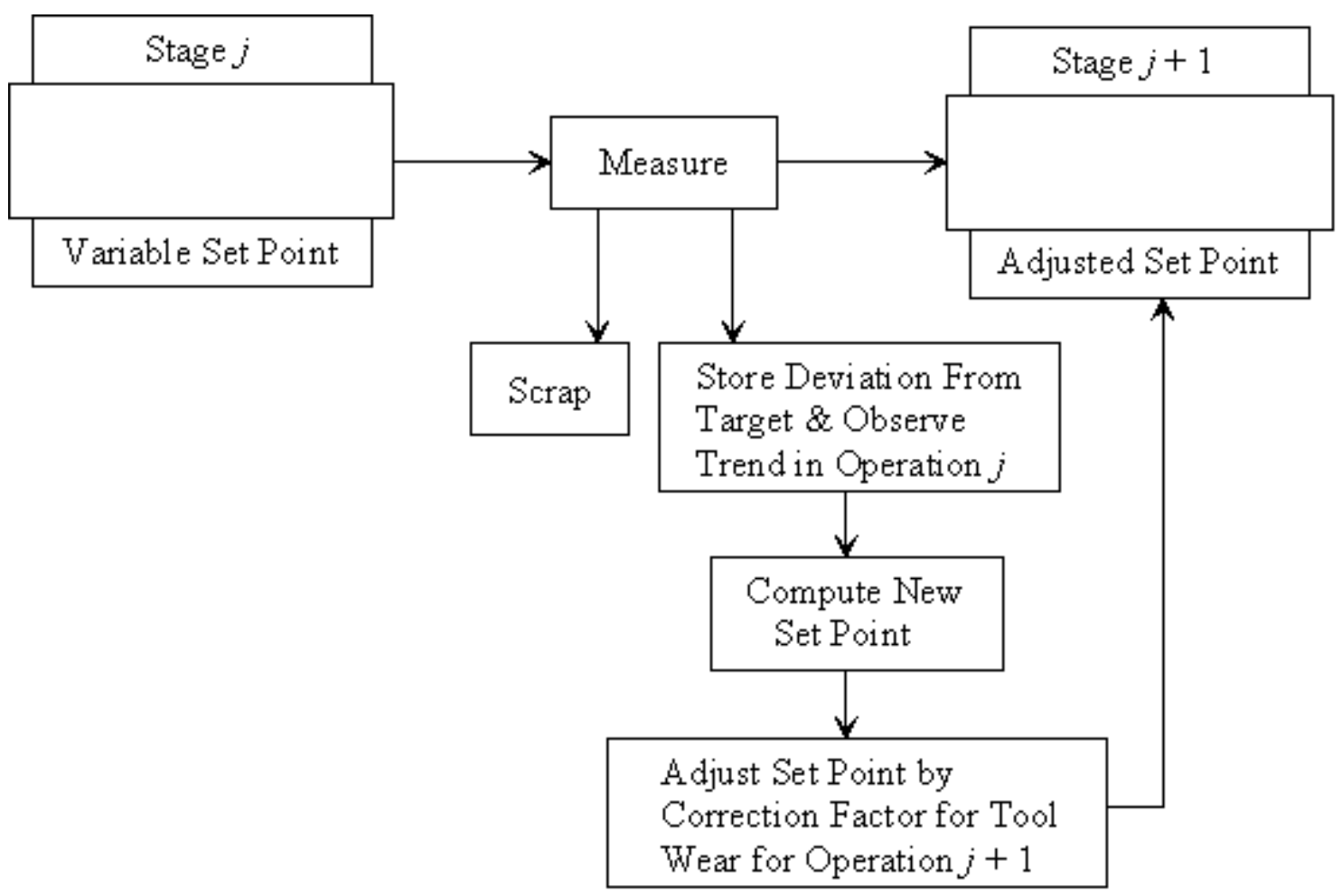

Figure 14. STC with Tool-wear Compensation

This concept can best be understood with a simple example. Consider again the hypothetical part in Figure 2 in which the relationship between dimensions $x_{1}, x_{2}, x_{3}$ and the constraints $c_{1}$ ,$c_{2}$, and $c_{3}$ is given in (1). As noted earlier, the conventional approach would select the fixed set point $\left(x_{1}, x_{2}, x_{3}\right)^{\mathrm{t}}=(2.250,1.750,1.250)^{\mathrm{t}}$ with allowable tolerances $\left(\delta_{1}, \delta_{2}, \delta_{3}\right)^{\mathrm{t}}=( \pm 0.0005$, $\pm 0.001, \pm 0.0005)^{\mathrm{t}}$, where $\delta_{i}$ is the tolerance range of each operation. 
Figure 15 assumes a realization of $x_{1}=2.2505$ and shows the resulting feasible domain of operations $x_{2}$ and $x_{3}$ plotted relative to initial set point $\mathbf{o}_{1}$. If set point $\mathbf{o}_{1}$ were kept for the next operations, a deviation for operation $x_{3}$ in excess of 0.0005 in the negative direction would produce a bad part. STC would then probably move the new set point to $\mathbf{0}_{2}$ for operations $x_{2}$ and $x_{3}$. Suppose, however, that it was inferred that the effect of tool wear would cause a deviation in the negative direction of 0.0011 from the target value of operation 2. Then, setting the target to $\mathbf{o}_{2}$ would most likely move the mean value to $\mathbf{d}$, which is close to the border of the feasible space. Therefore, in order to move the produced mean to the value of $\mathbf{o}_{2}$, the target value would probably be set equal to $\mathbf{o}_{2}^{\prime}$. If the actual effect of tool wear is close to the anticipated value, then the actual set point would be fairly well-centered in the feasible region, so that it could best compensate for the random deviation in operations 2 and 3.

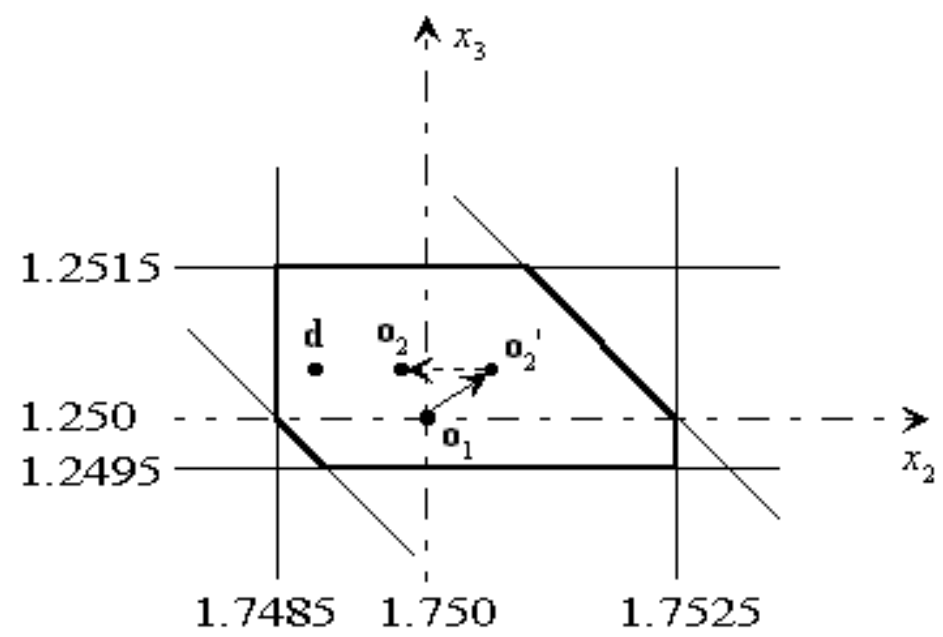

Figure 15. Resulting Feasible Domain when $x_{1}=2.2505$

Possible Tool-Wear Correction Factors: In the development of specific correction factors, some new notation is introduced. The correction factor for operation $i$ on part number $j$ is denoted as $\Delta_{i j}$. Denoting $x_{i j \text { target }}$ as the target value for operation $i$ on part $j$ and $\hat{x}_{i j}$ as the actual output of that operation, the deviation of the actual process output from its target is denoted as $d e v_{i j}=\hat{x}_{i j}-x_{i j \text { target }}$. Note that during the production of the first several parts, random deviations could occur in such a way that inferences about the actual effect of tool wear are unclear or even inaccurate. For this reason, although information about process outputs is stored for the first several parts, correction factors are not immediately applied. The number of parts to produce before applying the correction factor should be determined by the process planner.

Although the values of $d e v_{i j}$ give an accurate representation of the deviation from the target value for the parts that are produced before a correction factor is applied, they do not reveal any systematic variations from the target value once a correction factor has been applied. Consider, for example, that the effect of tool wear has caused the output of operation $i$ to be 
systematically larger than its target value for the first $j$ - 1 parts produced without applying a correction factor. If an accurate correction factor is used for operation $i$ on part $j$, the correction factor, $\Delta_{i j}$, will infer and compensate for the systematic deviation due to tool wear. If $\Delta_{i j}$ completely compensates for the effect of tool wear, then $d e v_{i j}$ will be equal to the random deviation only. If this value, $d e v_{i j}$, were used to infer a tool-wear trend for future parts, it would appear that no effect of tool wear was present. In effect, the accuracy of the correction factors, $\Delta_{i j}$, would actually prevent accurate corrections in future parts. In order to avoid this problem, the value $d e v_{i j}+\Delta_{i j}$ is recorded as the deviation to be analyzed for a toolwear trend. This value is equal to the deviation that would have occurred if no correction factor had been applied. It is precisely this deviation that reflects the true effect of tool wear.

The first proposed correction strategy calculates the least-squares linear regression line for the deviations for operation $i$ for all previous parts. The correction factor is determined by extrapolating the linear regression line to the next part and noting the forecasted deviation. This forecasted deviation is used as the correction factor when a Type I error associated with the regression line is smaller than a prescribed value, $p$. If the Type I error is larger than $p$, the correction factor is set equal to zero. That is, only when the slope of the regression line is found to be significantly different from zero is the correction factor applied. This prevents unnecessary corrections for tool wear if a trend in previous deviations is not clear. Given the slope, $\beta_{i 1}$, and the intercept, $\beta_{i 0}$, of the regression line calculated based on the deviations of the first $l-1$ parts, the tool-wear correction factor for operation $i$ on the current part number, $l$, is given by.

$$
\begin{aligned}
\Delta_{i l} & =\beta_{i 0}+\beta_{i 1}(l), & & \text { if Type I Error } \leq p \\
& =0, & & \text { Otherwise }
\end{aligned}
$$

The second strategy, known as the slope approximation strategy, is a simplification of the regression concept. The tool-wear regression line should theoretically have an intercept of zero, since the tool is assumed to be new at the start of production, indicating no effect due to tool wear. In addition, it can be assumed that the effect of tool wear would produce the average deviation value halfway through the production. Therefore, in the slope approximation approach, the intercept of the regression line is set equal to zero, and the slope is set equal to the average deviation divided by half of the number of parts produced to that time. Using the same notation as in the linear regression model, the intercept, $\beta_{i 0}$, is set equal to zero. The slope, $\beta_{i 1}$, is equal to

$$
\left(\frac{2 \sum_{j=1}^{l-1} d e v_{i j}}{(l-1)^{2}}\right)
$$

This line is projected to the current part, $l$, and the associated deviation is given as the correction factor, 


$$
\Delta_{i l}=\left(\frac{2 \sum_{j=1}^{l-1} d e v_{i j}}{(l-1)^{2}}\right)(l)
$$

In order to contrast these correction methods, a simple illustration is provided. Given that a total of nine parts have been produced, with part number 10 ready to start operation $i$. The deviations, $d e v_{i j}$, for operation $i$ with $j=1,2, \ldots, 9$ are displayed graphically in Figure 16. Note that these deviations are purely illustrative and are not to scale. The linear regression method would use the method of least-squares to determine a line with slope of 1.25 and an intercept of -1.36 units, indicating that the effect of tool wear for part 10 would be 11.14 units. The slope approximation method gives a slope of 1.086, indicating that the anticipated effect of tool wear for part 10 would be 10.86 units.

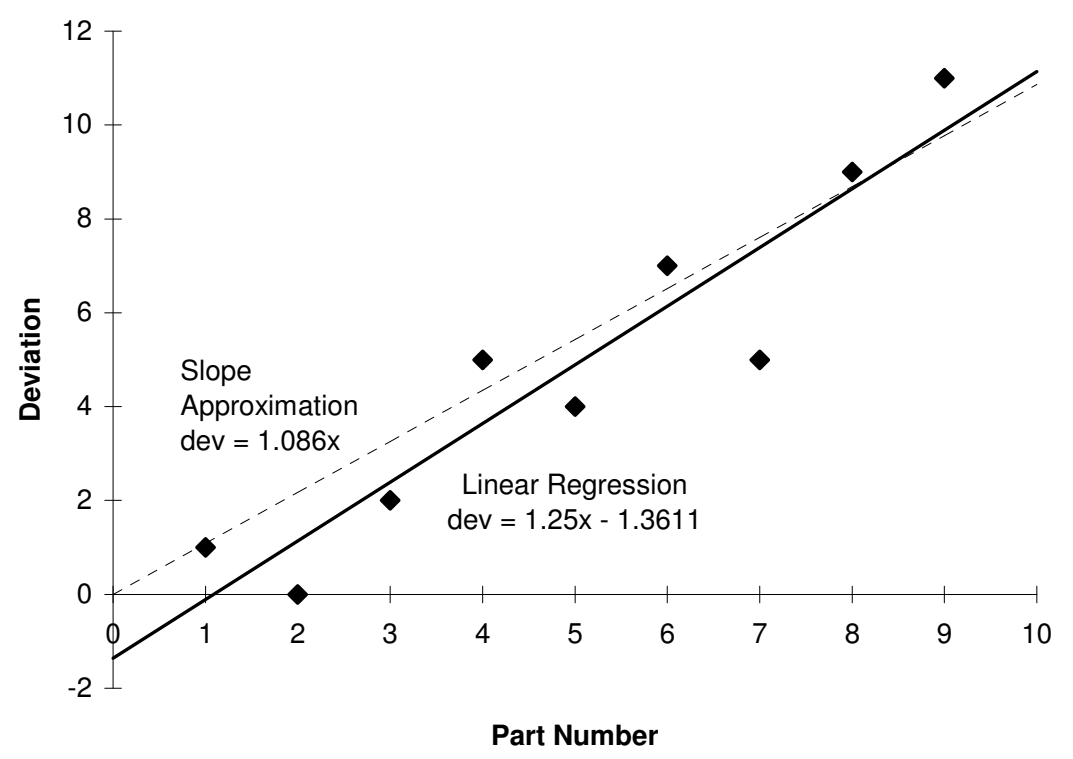

Figure 16. Comparison of Tool-wear Correction Methods

Implementation of Tool-Wear Compensation: In order to evaluate the performance of the two correction methods, each of the correction factors was simulated on the production of a cast steel drive hub. The simulation module, coded in FORTRAN, included a section that performed STC by solving the linear programming formulations (4) and (5), and another section simulated the effect of tool wear and storage of the process deviations. In addition, a section was added to compute and apply correction factors provided by each alternative. The manufacturing operations prescribed in the process plan were executed in the chronological order given by the plan. To execute a given manufacturing operation, the simulation module 
generates a random deviate from a uniform density function with a mean that drifts over time by a preset amount. Note that the selection of the uniform density function constitutes, in effect, a worst case scenario for the random deviates. The module computes the effect of tool wear as an incremental increase for each part produced. The same random deviate and tool wear component are then applied to the corresponding part produced by each of the studied correction methods. For each operation of each processed part, the simulation module tracked all constraints that are violated, and the total number of rejected parts was calculated for each of the correction factors.

The cast steel drive hub (Eary and Johnson [2]) shown in Figure 9 was again chosen as a test part. Recall that the constraints defining the problem are given in (6) and (7). These constraints were read into the simulation module from an input file.

It should be noted that the random variation of the simulated processes is smaller than the ideal variability that guarantees $100 \%$ acceptance in the non-tool-wear problem. In the simulation, the relationship between these quantities is represented by $\gamma$, the ratio between the simulated and ideal variability ranges. Additionally, over the entire length of production, the process mean is set to drift by a set multiple, $D_{t}$, of the range of the random deviations. That is, the mean value of the deviation was centered at zero for the first part, indicating that tool wear has not affected the output. For each part after that, however, the mean deviation shifted linearly until the mean for the last part is located at $D_{t}$ times the range of random deviations. The combined effect of tool wear and random variation is simulated by shifting the process mean linearly over time and by then applying a random component to the deviation. This concept is illustrated in Figure 17. In the figure, the value of $\gamma$ is equal 0.375 , indicating that the range of the random deviations is equal to three-eighths of the ideal variability range used in the non-tool-wear problem. When the process begins, the range of deviations is centered at zero, but by the end of production, the effect of tool wear has caused this range to shift. Note that the drift is most significant when $D_{t}$ is equal to 2 and 1.5 , since there is no possibility that the same total process deviation can be generated at the start and end of production. As $D_{t}$ decreases below 1, however, it is possible to have the same total deviations throughout production, indicating that the effect of tool wear is much less pronounced. 


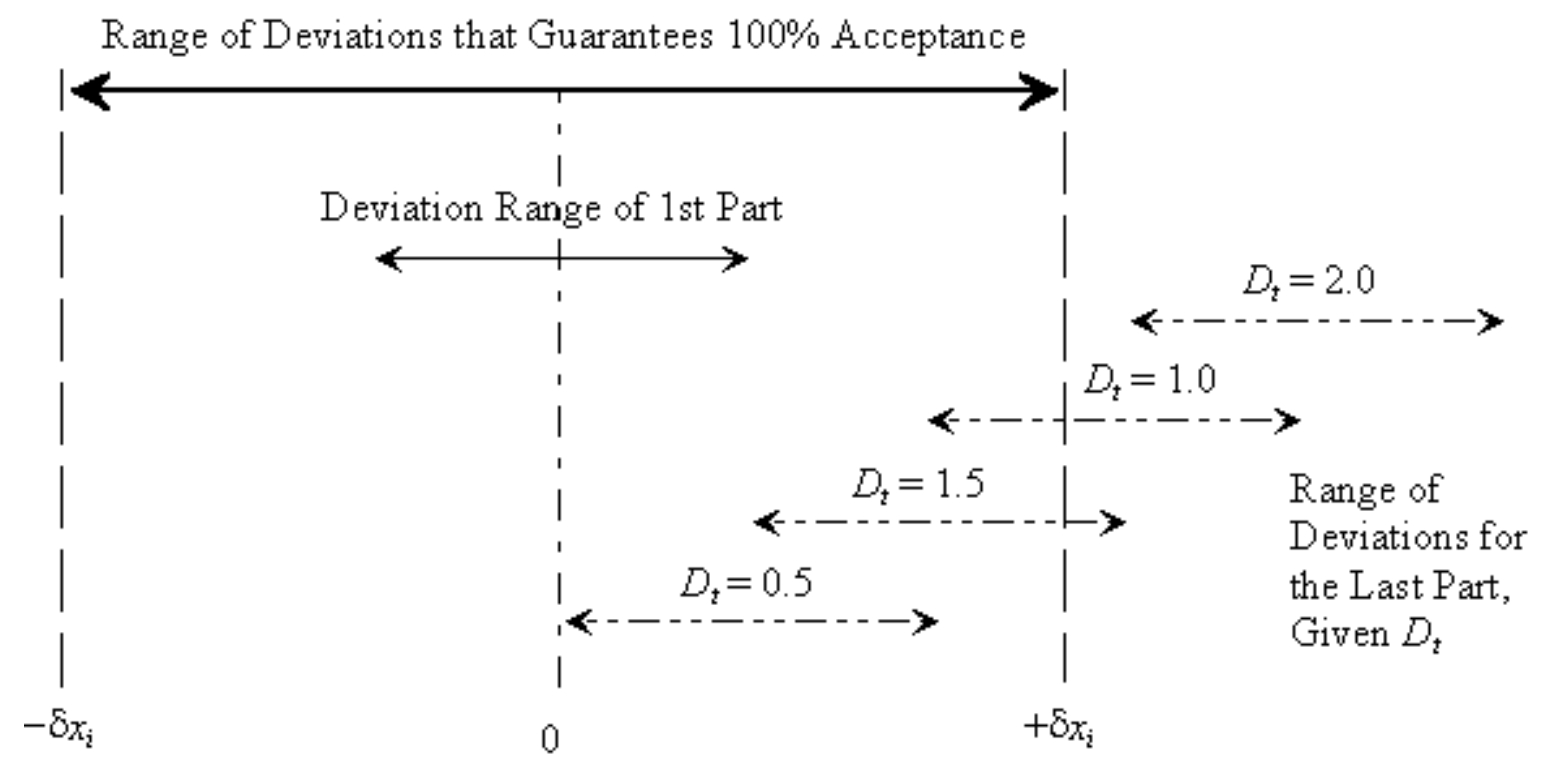

Figure 17. Ranges of Random Deviations

A summary of trial conditions is given in Table 2. Note that for each method, each of the four values of $D_{t}$ is simulated with each of the seven values of $\gamma$, for a total of 28 runs. For each of these runs, a total of five trials were performed, where each trial simulated the production of 50 parts. After these trials were evaluated, a subsequent run was performed in which the best correction factor was applied to the traditional tolerance control method for comparative purposes.

Table 2. Test Conditions

\begin{tabular}{|l|l|ll|l|}
\hline Method & Correction Factor & \multicolumn{2}{|c|}{$\gamma$} & $D_{t}$ \\
\hline Sequential Tolerance Control & None & 0.125 & 0.25 & 0.5 \\
& Linear Regression, $\mathrm{p}=0.1$ & 0.375 & 0.5 & 1.0 \\
& Linear Regression, $\mathrm{p}=0.2$ & 0.625 & 0.75 & 1.5 \\
& Slope Approximation & 1 & & 2.0 \\
\hline Traditional Tolerance Control & Slope Approximation & & & \\
\hline
\end{tabular}

Tool-Wear Compensation Simulation Results and Discussion: The results of the simulation are summarized in Table 3, which shows the average number of rejects (out of 50 parts produced) for each of the 28 combinations of $D_{t}$ and $\gamma$. Uncorrected STC performs dramatically worse than any of the corrected methods. The large number of defects for the uncorrected method demonstrates the inability of STC alone to compensate for drift. It is believed that the addition of a deterministic component of variation reduces the ability of the sequential method to re-center the process mean to a central portion of the feasible region, since re-centering can be nullified by the effect of tool wear. The adjusted set point may even 
prove worse than the traditional set point, if the adjustment combines with tool wear in an undesirable direction.

Since a correction factor for tool wear is clearly warranted, the focus now turns to which of the correction factors performs best. When $\gamma$ is less than or equal to 0.5 , the three methods completely correct for the effect of drift, producing no defective parts in any trial. For $\gamma=$ 0.625 and $\gamma=0.75$, the slope approximation method produces the smallest number of defective parts for each of the $D_{t}$ values. The only case in which the slope approximation method does not consistently out-perform the two linear regression methods is when $\gamma=1.0$. In this case, the three methods provide very similar results, with slope approximation producing the fewest defectives for three of the four $D_{t}$. values. In summary, slope approximation produces the smallest number of rejected parts in 27 of the 28 simulation runs. For this reason, it appears that a correction factor for tool wear is most appropriately determined by the slope approximation method.

A simulation was then performed to evaluate how this correction factor worked in conjunction with traditional tolerance control. These results are also displayed in Table 3.

Table 3. Number of Defective Parts for Corrected and Uncorrected Methods

\begin{tabular}{|c|c|c|c|c|c|c|}
\hline \multirow[t]{2}{*}{$\gamma$} & \multirow[t]{2}{*}{$D_{t}$} & \multicolumn{4}{|c|}{ Sequential Tolerance Control } & \multirow{2}{*}{$\begin{array}{l}\text { Conventional } \\
\text { Tolerance Control } \\
\text { Slope Approx. }\end{array}$} \\
\hline & & $\begin{array}{l}\text { Lin. Reg. } \\
p=0.1\end{array}$ & $\begin{array}{l}\text { Lin. Reg. } \\
p=0.2\end{array}$ & Slope Approx. & Uncorrected & \\
\hline \multirow[t]{4}{*}{0.125} & 2 & 0 & 0 & 0 & 0 & 0 \\
\hline & 1.5 & 0 & 0 & 0 & 0 & 0 \\
\hline & 1 & 0 & 0 & 0 & 0 & 0 \\
\hline & 0.5 & 0 & 0 & 0 & 0 & 0 \\
\hline \multirow[t]{4}{*}{0.25} & 2 & 0 & 0 & 0 & 0 & 0 \\
\hline & 1.5 & 0 & 0 & 0 & 0 & 0 \\
\hline & 1 & 0 & 0 & 0 & 0 & 0 \\
\hline & 0.5 & 0 & 0 & 0 & 0 & 0 \\
\hline \multirow[t]{4}{*}{0.375} & 2 & 0 & 0 & 0 & 11.6 & 0 \\
\hline & 1.5 & 0 & 0 & 0 & 2.4 & 0 \\
\hline & 1 & 0 & 0 & 0 & 0 & 0 \\
\hline & 0.5 & 0 & 0 & 0 & 0 & 0 \\
\hline \multirow[t]{4}{*}{0.5} & 2 & 0 & 0 & 0 & 21.2 & 0 \\
\hline & 1.5 & 0 & 0 & 0 & 13.2 & 0 \\
\hline & 1 & 0 & 0 & 0 & 2.2 & 0 \\
\hline & 0.5 & 0 & 0 & 0 & 0 & 0 \\
\hline \multirow[t]{3}{*}{0.625} & 2 & 0 & 0 & 0 & 27.8 & 0 \\
\hline & 1.5 & 0.2 & 0.2 & 0 & 21.2 & 0 \\
\hline & 1 & 0 & 0 & 0 & 9.4 & 0 \\
\hline
\end{tabular}




\begin{tabular}{|c|c|c|c|c|c|c|}
\cline { 2 - 7 } \multicolumn{1}{c|}{} & 0.5 & 0 & 0 & 0 & 0 & 0 \\
\hline 0.75 & 2 & 1.6 & 1 & 0.2 & 34 & 0.2 \\
\cline { 2 - 7 } & 1.5 & 1 & 0.6 & 0 & 27.4 & 0 \\
\cline { 2 - 7 } & 1 & 1 & 0.6 & 0 & 15.6 & 0 \\
\cline { 2 - 7 } & 0.5 & 0.4 & 0.4 & 0 & 1.6 & 0 \\
\hline \multirow{3}{*}{1} & 2 & 4.8 & 4.2 & 4.6 & 39.4 & 7 \\
\cline { 2 - 7 } & 1.5 & 5 & 4.6 & 4.4 & 36.6 & 6.2 \\
\cline { 2 - 7 } & 1 & 5.6 & 4 & 4 & 27.8 & 6 \\
\cline { 2 - 7 } & 0.5 & 4.6 & 4 & 3.6 & 10.6 & 5.6 \\
\hline
\end{tabular}

The slope approximation correction factor also performs well on the traditional method. For cases where $\gamma$ is less than one, the traditional and sequential methods produce an equal number of defective parts. When the value of $\gamma$ is increased to one, however, the sequential method out-performs the traditional method. With $D_{t}$ equal to $2,1.5,1$, and 0.5 , the sequential method reduces the number of defective parts by $34.3 \%, 29.0 \%, 33.3 \%$, and $35.8 \%$, respectively, from the number produced by the traditional method. This trend is not surprising, though, since the correction factor deals with the deterministic variation. If this deterministic variation could be totally eliminated, the random deviation of the process would always be centered at zero, indicating that neither traditional nor sequential tolerance control should produce defective parts when $\gamma$ is less than one. When systematic variation combines with less precise processes, however, the advantage of STC becomes clear. It therefore seems clear that when the effect of tool wear is present, the optimal policy is to employ STC with slope approximation correction factors.

Implicit Enumeration Approach to Tolerance Allocation in STC: One of the main activities performed by process planning in discrete parts manufacturing involves the development of a logical and chronological sequence of metal removal operations. These operations are designed to transform a raw material shape into a finished material shape compatible with the specified design dimensioning and tolerancing scheme. Execution of this sequence of operations results in the simultaneous or sequential creation of surfaces with associated measurable manufactured dimensions and tolerances. Each design dimensioning and tolerancing constraint is ultimately satisfied at a level dependent on the accuracy and precision of a particular subset of measurable manufactured dimensions. The accuracy and precision of a manufactured dimension is in turn dependent to a large extent on the accuracy and precision of the technological means or processes selected for its execution. A technological process in this context includes the machine tool, cutting tool, fixture, and all other human and environmental conditions prevailing during execution of the operation. It is not unusual for process planners to have available several competing technological processes for execution of each manufacturing operation specified in the process plan. Each candidate process offers a particular level of precision (tolerance range) and associated cost. Selection of an appropriate subset of technological processes to execute the set of manufacturing operations prescribed in a process plan is an important process planning activity. This selection is often constrained by considerations of process cost and yield subject to the generally accepted notion that as the precision of the technological process improves, the 
associated cost and yield of the operation increase. This paper presents an implicit enumeration approach to the selection of an optimum subset of technological processes required to execute a process plan under a sequential (or adaptive) tolerance control strategy.

As mentioned earlier, selection of the precision required of technological processes under a conventional tolerance control strategy is commonly known as the tolerance chart allocation problem ([1] - [16]). The tolerance allocation (synthesis) problem in design has also been treated extensively in the literature both deterministically and stochastically ([28] - [50]). Of particular interest is the paper by Lee and Woo [48] that develops an implicit enumeration approach to the allocation of discrete tolerances for a design problem. The formulation is a nonlinear 0-1 integer programming problem which seeks to minimize cost subject to constraints on the individual yield of each functional requirement. This formulation avoids the difficult task of computing the true yield in the presence of non-independent functional requirements and the algorithm returns an optimum solution with the knowledge that each individual functional requirement is independently satisfied with at least a given probability.

A similar difficulty is encountered in our attempt to treat tolerance allocation under STC. Whereas conventional tolerance control uses the nominal value from design specifications as the target value for each operation, STC uses measurement information acquired at the completion of operation $j$ to selectively adjust the set point of the remaining $(n-j)$ operations in the sequence. Wheeler et al [51] developed an implicit enumeration optimization strategy that uses simulation to compute the yield of the overall sequence given stochastic and dynamic adjustment conditions prevailing under STC. The methodology presented here is due to Wheeler et al [51].

Consider the problem of selecting an optimal set of processes when several candidate processes are available for each required operation of the process plan. An explicit enumeration of all possible combinations is an obvious way to proceed. However, for a typical part with 9 operations and 4 candidate processes per operation, yield computations will require $4^{9}=262,144$ possible process combinations to be simulated. A more efficient method than total enumeration is therefore desirable, and such a method is described below.

The mechanics of such a search will be demonstrated by considering the processing of a small part. Suppose that 3 operations are required to produce the part, and each operation has 4 processes from which to choose. The processes for each operation are numbered from 0 to 3 . Process 0 will be the most precise and therefore the most expensive process while process 3 will be the least precise and least expensive process. Let the triplet $(i j k)$ indicate that process $i$ is used for operation 1 , process $j$ for operation 2 , and process $k$ for operation 3 .

The search for the optimal process combination is made more efficient by organizing process combinations into a tree that exploits the monotone decreasing operating cost as the process precision deteriorates. In addition, the monotone decreasing probability of producing an acceptable part as a function of deteriorating process precision can also be exploited. The tree for this example is shown in Figure 18. 
Note that the tree has the same number of levels as there are operations to perform. Each node in the tree will be designated by a triplet using the notation described above. Nodes for the first level are obtained by fixing the most precise process for operations 2 and 3 while increasing the process choice for operation 1 . There will be 4 level-1 nodes, one node for each process for operation 1 . The children of a level-1 node can be obtained by fixing processes for operations 1 and 3 from the parent while assigning a process for operation 2 to each child. Each node at the first level will have 4 children since operation 2 has four processes to choose from. Each level-2 node will also have 4 children. To obtain level-3 nodes, fix the processes for operations 1 and 2 and assign a process for operation 3 to each child. 


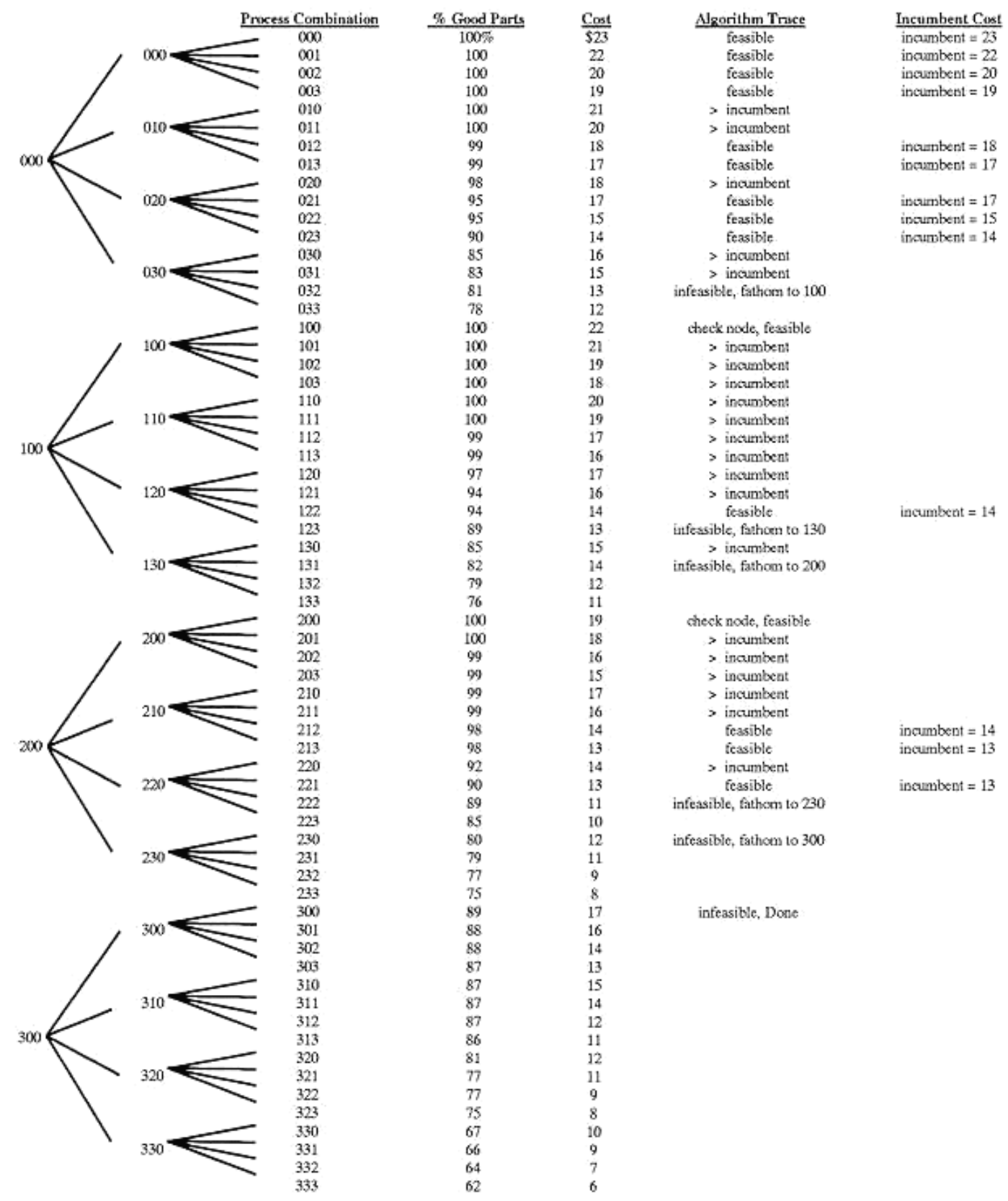

Figure 18. Explicit Enumeration of the Node Tree Generated by the 3-Operation Part and the Trace of the Implicit Enumeration Algorithm

Level-3 nodes represent all possible process combinations for the part. In general, if there are $n$ operations, level- $n$ will contain all possible combinations. For this reason it will be sufficient to search the bottom level of the tree to determine the optimal process combination. The cost of each node is readily determined by summing the individual process costs. The 
probability of an acceptable part is determined via Monte Carlo simulation that generates a vector of random deviates using appropriate density functions and then checks the location of the vector relative to the feasible space. Explicit enumeration of the 64 process combinations for this example would not be overly time consuming. However, since each examined node requires a simulation to estimate yield, explicit enumeration of the tree representing more complex parts can be prohibitive.

The triplet that designates a node in this example is a base 4 number since the highest digit used in any triplet is 3 . In general, nodes will be designated by a number in base $b$, where $b$ is the maximum number of processes available to any operation. The idea of thinking of a triplet as a base $b$ number will be used as a search index since level-3 nodes are an ordered sequence of these triplets. Node designations are converted into decimal numbers in order to implement the search on a computer.

Another useful characteristic of the process combination tree is that level-3 nodes can be further distinguished by the level in which they first appear. For example, node (100) appears first in level 1, however it also is present in levels 2 and 3. Node (100) will be referred to as a level-1 node. Node (010) first appears in level 2 and will thus be referred to as a level-2 node. The number of trailing zeros in a node designation will indicate the level of that node. If $n$ operations are required and the node has $k$ trailing zeros then that node will be from level $(n-k)$.

It is useful to note that, in this example, if node designations are converted into decimal numbers, then all level- 1 nodes are divisible by $4^{2}=16$. In addition, all level- 2 nodes are divisible by 4 . In general, if $n$ operations are required and there are at most $b$ processes for each operation, then level-1 nodes (after converting to decimal) will be divisible by $b^{n-1}$. Level-2 nodes will be divisible by $b^{n-2}$, level-3 nodes will be divisible by $b^{n-3}$, and level- $q$ nodes will be divisible by $b^{n-q}$. In addition, a level- $q$ node will have $b^{n-q}$ descendants. These observations constitute the key ideas used to fathom the tree when searching for the optimal process combination. The two-part fathoming procedure will now be described in more detail.

The probability of producing an acceptable part is a function of the precision of the processes used to produce that part. As the precision of any process deteriorates while all other processes are held constant, the probability of producing an acceptable part is nonincreasing. This is demonstrated on the process combination tree. Consider node (231) on the tree. As the precision of the process used to perform operation 3 deteriorates [i.e. moving from node (231) to (232) to (233)] the probability of producing an acceptable part decreases from $79 \%$ to $77 \%$ to $75 \%$.

This function is guaranteed to decrease only until a node from a higher level is encountered. In this case, when we move from (233) to (300) [(300) is a level-1 node since it first appears in level 1] the probability of producing an acceptable part increases from $75 \%$ to $89 \%$. Therefore, if it is determined that the probability of producing an acceptable part is below the minimum level, then it is not necessary to consider any subsequent process combinations 
until a combination which appears at a higher level is encountered. This basic idea is formalized as the following proposition.

Proposition 1: If a combination is infeasible then that combinations siblings and their descendants that have not yet been examined will also be infeasible and can be fathomed from the tree. That is, if process combination $p_{1} p_{2} \ldots p_{k-1} p_{k} p_{k+1} \ldots p_{n}$ is infeasible (i.e. it does not produce an acceptable percentage of good parts), then all combinations $p_{1} p_{2} \ldots p_{k-1}$ $t p_{k+1} \ldots p_{n}$ for $t \geq p_{k}$, can be fathomed from the tree.

This follows from the fact that increasing the precision of one process while holding all other processes constant cannot cause a decrease in the proportion of acceptable parts. When an infeasible node is encountered, the algorithm should skip all nodes until a node from a higher level is encountered. The method of determining the number of nodes that need not be considered is presented next.

If a node is determined to be infeasible then the search should skip to the next higher-level node. That is, skip all of the current node's siblings that have not yet been considered and their descendants. To determine the number of nodes to skip simply multiply the number of remaining siblings, including the current node, by the number of descendants of each node at this level.

As an example, consider a 9-operation part with 4 processes available to each operation and an infeasible level-6 node (233122000). Node (233122000) has 1 sibling (233123000) and each level-6 node has $b^{n-6}=4^{9-6}=64$ descendants. The algorithm will skip the current node's descendants as well as its sibling's descendants. That is, the next 128 nodes need not be considered for simulation. In general, if a level- $q$ node $\left(p_{1} p_{2} \ldots p_{q-1} t 0 \ldots\right)$ is determined to be infeasible with process $t$ used for operation $q$ then $(b-t) b^{n-q}$ nodes should be skipped, where $(b-t)$ is the number of remaining siblings plus the current node.

The implicit enumeration algorithm can be made more efficient by further exploiting Proposition 1. If any level-1 node is determined to be infeasible after simulation, then from Proposition 1 all remaining process combinations can be fathomed. If a level- 2 node is infeasible then all nodes can be fathomed until a level-1 node is encountered. Therefore complex parts allow for many nodes to be fathomed if a level-1 or level-2 node is determined to be infeasible. However, a process combination will be simulated only if its associated cost is less than or equal to the incumbent (or current best) cost. Let a check node be a node that will be simulated regardless of its associated cost in order to maximize the number of fathomed process combinations. The set of nodes designated as check nodes will vary with the number of operations required to produce the part. Consider, for example, the 9operation part described above. It may be determined that all level-4 nodes will be check nodes. It would be inefficient to designate lower level nodes (i.e. level 5, level 6,...) as check nodes because the additional simulation of nodes regardless of cost would be greater than the savings realized from fathoming due to infeasibility. The concept of a check node will be further discussed in the context of the examples, which will be presented subsequently. 
The second method of fathoming the tree will be based on the cost associated with a process combination. As we move across the bottom level of the tree, many nodes will not be simulated due to the fathoming described above. If it is determined that a node should not be skipped, then the cost of the current node will be compared with the incumbent cost associated with an acceptable node. If the cost of the current node is lower than the incumbent cost then the process combination will be simulated. Otherwise the search will move to the next node in the sequence. Fathoming due to cost consideration can be stated as follows

Proposition 2: If the cost of process combination $p_{1} p_{2} \ldots p_{k-1} p_{k} p_{k+1} \ldots p_{n}$ is greater than the incumbent cost, then this node should only be simulated if it is a check node. Otherwise the next combination to be considered will be combination $p_{1} p_{2} \ldots p_{k-1} p_{k} p_{k+1} \ldots p_{n}+1$. That is, consider the next node in the sequence.

Yield estimation for the implicit enumeration algorithm was carried out by simulation of the sequential and chronological execution of each prescribed operation. Starting with the first operation, a random deviate is generated from an appropriate probability density function having a range equal to the precision capability of the process selected for execution of that particular operation. The appropriate density function should be extracted from historical data. If historical data is not available, a rectangular distribution can be used to model the worst case conditions. The constraint set of the problem is then modified by inserting the actual value of the operation just executed. If the feasible zone is not empty, the linear programming problems [(4) and (5)] are solved to determine the new set point for the next operation. The sampling process, constraint update, and the set point adjustment are repeated until all operations are executed. A part is defective if the feasible zone becomes empty at any stage of the execution. Yield estimates were based on sample sizes of 1000 parts.

This implicit enumeration algorithm allows for alternative optimal solutions. This will occur if two or more sets of processes satisfy the minimum proportion of good parts while minimizing manufacturing costs. The knowledge that there exist multiple optimal process combinations can be utilized to allow for flexibility in scheduling the processes. The algorithm used in the implicit enumeration method is described in the flowchart shown in Figure 19. 


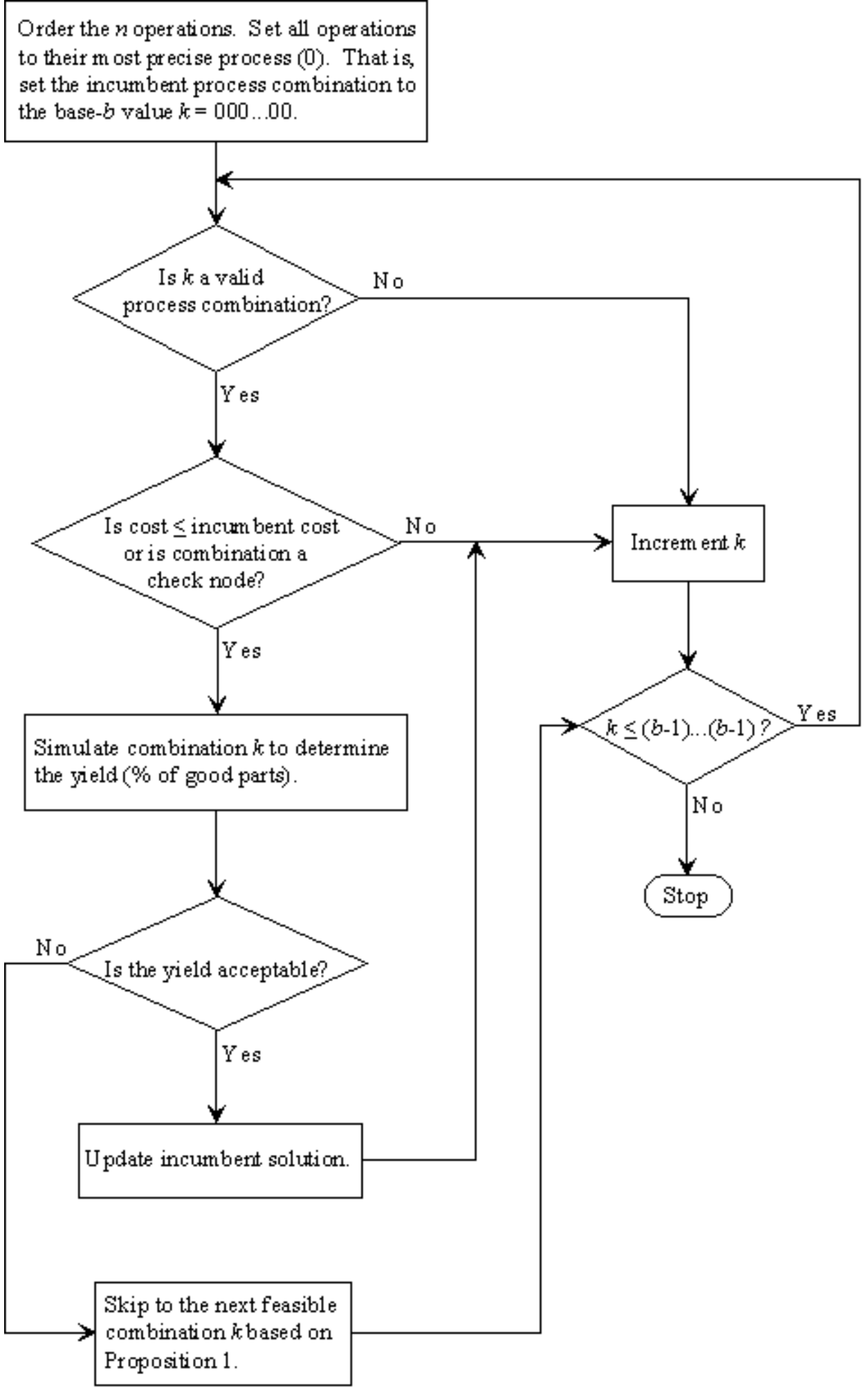

Figure 19. Flowchart for the Implicit Enumeration Procedure 
Implicit Enumeration - Examples and Computational Results: Solution of a 3-Operation Part: A detailed solution to the 3-operation part depicted in Figure 2 will now be presented. As previously noted, the relationship between the dimensions $x_{1}, x_{2}, x_{3}$ produced by manufacturing operations $X_{1}, X_{2}$, and $X_{3}$ and the constraints $c_{1}, c_{2}, c_{3}$ are given by the system of equations provided in (1). The capability and cost of each process for the three operations are described in Table 4.

Table 4. Capability and Costs for Processes Available for the 3-Operation Part

\begin{tabular}{|c|c|c|c|c|c|c|}
\hline & \multicolumn{2}{|c|}{ Operation 1 $\left(\boldsymbol{x}_{\mathbf{1}}\right)$} & \multicolumn{2}{c|}{ Operation 2 $\left(\boldsymbol{x}_{\mathbf{2}}\right)$} & \multicolumn{2}{c|}{ Operation 3 $\left(\boldsymbol{x}_{\mathbf{3}}\right)$} \\
Process \# & Precision & Cost & Precision & Cost & Precision & Cost \\
\hline 0 & 0.0050 & 7 & 0.0010 & 9 & 0.0005 & 7 \\
1 & 0.0070 & 6 & 0.0014 & 7 & 0.0007 & 6 \\
2 & 0.0090 & 3 & 0.0018 & 4 & 0.0020 & 4 \\
3 & 0.0110 & 1 & 0.0022 & 2 & 0.0022 & 3 \\
\hline
\end{tabular}

Figure 18 depicts an explicit enumeration of all 64 possible process combinations along with their associated costs and proportion of acceptable parts. For the purpose of illustration the minimum proportion of acceptable parts for this example is set at 90\%. All level-1 nodes will be check nodes. The utility of the check nodes is clearly demonstrated in this example. If node (300) had not been a check node, the algorithm would not have simulated the combination since the associated cost of $\$ 17$ is greater than the incumbent cost. However, since it is a check node, it was simulated and was determined to be infeasible. Therefore $25 \%$ of the process combinations were fathomed resulting in a substantial savings in time.

As can be seen from the solution, there are 2 process combinations that yield an acceptable proportion of good parts at minimum cost. The fact that there exist alternative optimal process combinations will be useful for process scheduling purposes. Depending on the availability of processes, either combination (213) or (221) could be used. Note, however, that there is a substantial difference in the proportion of good parts. Combination (213) yields $98 \%$ good parts as compared to $90 \%$ from combination (221). Even though both exceed the minimum level, this difference may be a consideration in the scheduling process if the cost of additional defects is significant.

Solution of a 10-Operation Problem: In order to demonstrate the efficiency of this algorithm, the tolerance allocation problem associated with the part in Figure 9 was solved. The part requires 9 operations (Note that $x_{7}=x_{8}$.) with 4 processes available for each operation. Table 5 shows the precision capability of each process along with its associated cost factor. The dimension produced by each operation is also indicated. That is, dimension $x_{10}$ is produced using the process choice specified in operation 1. The assignment of a dimension to an operation was done arbitrarily for this problem. The assignment of a dimension to an operation simply specifies where in the looping process the process choice is varied. The algorithm changes the process for operation 9 the most rapidly whereas the process for operation 1 is changed the slowest. That is, the algorithm is a 9-level nested loop with the process for operation 1 specified by the outermost loop. The process for operation 9 is 
specified by the innermost loop. Further research is being conducted to determine the most efficient ordering of the dimensions. The structure of the design and process planning constraints will be examined to determine the optimal ordering of dimensions needed to fathom the tree most efficiently.

Table 5. Capability and costs for processes available for the 10-operation part

\begin{tabular}{|c|c|c|c|c|c|c|}
\hline & \multicolumn{2}{|c|}{ Operation 1 $\left(\boldsymbol{x}_{\mathbf{1 0}}\right)$} & \multicolumn{2}{c|}{ Operation 2 $\left(\boldsymbol{x}_{\mathbf{9}}\right)$} & \multicolumn{2}{c|}{ Operation 3 $\left(\boldsymbol{x}_{\mathbf{7}} / \boldsymbol{x}_{\mathbf{8}}\right)$} \\
Process \# & Precision & Cost & Precision & Cost & Precision & Cost \\
\hline 0 & 0.0010 & 9 & 0.0010 & 7 & 0.0070 & 8 \\
1 & 0.0014 & 7 & 0.0014 & 5 & 0.0098 & 7 \\
2 & 0.0018 & 6 & 0.0018 & 4 & 0.0126 & 6 \\
3 & 0.0022 & 4 & 0.0022 & 3 & 0.0154 & 5 \\
\hline
\end{tabular}

\begin{tabular}{|c|c|c|c|c|c|c|}
\hline & \multicolumn{2}{|c|}{ Operation $4\left(\boldsymbol{x}_{\mathbf{6}}\right)$} & \multicolumn{2}{c|}{ Operation 5 $\left(\boldsymbol{x}_{\mathbf{4}}\right)$} & \multicolumn{2}{c|}{ Operation 6 $\left(\boldsymbol{x}_{\mathbf{3}}\right)$} \\
Process \# & Precision & Cost & Precision & Cost & Precision & Cost \\
\hline 0 & 0.0030 & 7 & 0.0040 & 9 & 0.0020 & 8 \\
1 & 0.0042 & 5 & 0.0056 & 8 & 0.0028 & 5 \\
2 & 0.0054 & 4 & 0.0072 & 7 & 0.0036 & 4 \\
3 & 0.0066 & 3 & 0.0088 & 6 & 0.0044 & 3 \\
\hline
\end{tabular}

\begin{tabular}{|c|c|c|c|c|c|c|}
\hline & \multicolumn{2}{|c|}{ Operation 7 $\left(\boldsymbol{x}_{\mathbf{2}}\right)$} & \multicolumn{2}{c|}{ Operation 8 $\left(\boldsymbol{x}_{\mathbf{1}}\right)$} & \multicolumn{2}{c|}{ Operation 9 $(\boldsymbol{L})$} \\
Process \# & Precision & Cost & Precision & Cost & Precision & Cost \\
\hline 0 & 0.0020 & 7 & 0.0020 & 8 & 0.0020 & 8 \\
1 & 0.0028 & 5 & 0.0028 & 7 & 0.0028 & 7 \\
2 & 0.0036 & 4 & 0.0036 & 5 & 0.0036 & 6 \\
3 & 0.0044 & 3 & 0.0044 & 4 & 0.0044 & 4 \\
\hline
\end{tabular}

The optimal process combination was determined using a range of values for the minimum acceptable proportion of good parts. One thousand parts were simulated in order to determine the proportion of good parts corresponding to a process combination.

As can be seen from Table 6, STC enables the part to be produced at a substantially lower cost than can be realized with conventional tolerance control. This reflects the ability of STC to utilize the results of previous operations to compute new set points for subsequent operations such that the probability of producing a good part is maximized. The simulation of parts within STC utilizes a vector of random deviates to model actual operations. The random deviates were drawn from a uniform distribution with a range corresponding to the precision capability of the process. Use of a uniform distribution will lead to a higher proportion of rejected parts since in reality manufactured dimensions will in general exhibit density functions with decreasing tail probabilities.

Table 6. Implicit Enumeration Computational Results 


\begin{tabular}{|c|c|c|c|c|c|}
\hline \multirow[b]{2}{*}{$\begin{array}{c}\% \text { Good } \\
\text { Parts }\end{array}$} & \multicolumn{2}{|c|}{$\begin{array}{c}\text { Sequential Tolerance } \\
\text { Control }\end{array}$} & \multicolumn{2}{|c|}{$\begin{array}{c}\text { Conventional Tolerance } \\
\text { Control }\end{array}$} & \multirow[b]{2}{*}{$\begin{array}{l}\% \text { Cost } \\
\text { Savings }\end{array}$} \\
\hline & $\begin{array}{c}\text { Optimal } \\
\text { Cost }\end{array}$ & $\begin{array}{c}\text { \# of } \\
\text { Simulations }\end{array}$ & Optimal Cost & $\begin{array}{c}\text { \# of } \\
\text { Simulations }\end{array}$ & \\
\hline $99.5 \%$ & 52 & 44 & 63 & 64 & $17.5 \%$ \\
\hline $99 \%$ & 51 & 41 & 60 & 48 & $15.0 \%$ \\
\hline $98 \%$ & 50 & 45 & 58 & 81 & $13.7 \%$ \\
\hline $97 \%$ & 50 & 52 & 58 & 101 & $13.7 \%$ \\
\hline $96 \%$ & 50 & 61 & 56 & 147 & $10.7 \%$ \\
\hline $95 \%$ & 50 & 65 & 56 & 126 & $10.7 \%$ \\
\hline
\end{tabular}

Tolerance Allocation and Yield Computation using FOSMM: Most approaches to the probabilistic tolerance chart allocation problem found in the literature suffer from the inability to compute the total yield for the system of interrelated tolerance chains that describe the problem. The development presented here is due to Wheeler et al [53], who overcame this limitation by using the First Order Second Moment Method (FOSMM) (See [43], [44], [45], [48], [53], [54]) to compute system yield.

The formulation of the conventional tolerance chart problem is based on the representation of $p$ design and process planning constraints as a linear constraint set $c_{i} \in\left[c_{i \min }, c_{i \max }\right], i=$ $1, \ldots, p$ along a particular direction. This constraint set is to be satisfied by a combination of $n$ manufacturing operations $x_{j}, j=1, \ldots, n$ which combine in $p$ linear subsets to satisfy the constraint set. In general, the solution domain is a convex set represented by a set of linear inequalities,

$$
c_{i \min } \leq \mathbf{a}_{i} \mathbf{x}=\sum_{j=1}^{n} a_{i j} x_{j} \leq c_{i \max } \quad i=1, \ldots, p
$$

where $\mathbf{x}=\left(x_{1}, x_{2}, \ldots, x_{n}\right)^{\mathrm{t}}$ is the vector of manufacturing operations. In the probabilistic approach to the problem, $\mathbf{x}$ is now a vector of stochastic variables with known density functions and the task is to fix the permissible variability of each stochastic variable to satisfy a given objective function. A fairly common objective is to decide on an allocation that minimizes cost and meets a prescribed yield or acceptable fraction $(A F)$ of good parts. A good part in this context is one that meets all design and process planning constraints. FOSMM is used to enable computation of the yield or $A F$ associated with the set of interrelated tolerance chains described by (8).

Let each of the $p$ linear constraints given by (8) be rewritten as a pair of constraints in the following common format:

$$
\begin{aligned}
\mathbf{a}_{i} \mathbf{x}-c_{i \min } & =\sum_{j=1}^{n} a_{i j} x_{j}-c_{i \min } \geq 0 \\
-\mathbf{a}_{i} \mathbf{x}+c_{i \max } & =-\sum_{j=1}^{n} a_{i j} x_{j}+c_{i \max } \geq 0
\end{aligned}
$$


This representation gives rise to $m=2 p$ inequalities that can be represented in the general form:

$$
g_{i}(\mathbf{x}) \geq 0 \quad i=1, \ldots, m
$$

Each functional requirement $g_{i}(\mathbf{x}) \geq 0$ divides $n$-dimensional space into a failure set where $g_{i}(\mathbf{x})<0$ and an acceptable set where $g_{i}(\mathbf{x}) \geq 0$. The yield or acceptable fraction $(A F)$ is then given by

$$
A F=1-\operatorname{Pr}\{F\}=1-\operatorname{Pr}\left\{\bigcup_{i=1}^{m} F_{i}\right\}
$$

where

$$
\begin{aligned}
& \operatorname{Pr}\{F\}=\text { system failure probability } \\
& F_{i}=\text { event that } g_{i}(\mathbf{x})<0
\end{aligned}
$$

For a series system of $m$ such requirements, $\operatorname{Pr}\{F\}$ can be written as (Melchers [55])

$$
\operatorname{Pr}\{F\}=\sum_{i=1}^{m} \operatorname{Pr}\left\{F_{i}\right\}-\sum_{i=1}^{m} \sum_{\substack{j=1 \\ j>i}}^{m} \operatorname{Pr}\left\{F_{i} \cap F_{j}\right\}+\sum_{i=1}^{m} \sum_{\substack{j=1 \\ j>i}}^{m} \sum_{k=1}^{m} \operatorname{Pr}\left\{F_{i} \cap F_{j} \cap F_{k}\right\}-\ldots
$$

When $m$ is large, evaluation of (9) is impractical and a conservative estimate due to Ditlevsen [56] is given by:

$$
\operatorname{Pr}\{F\} \leq \sum_{i=1}^{m} \operatorname{Pr}\left\{F_{i}\right\}-\sum_{i=2}^{m} \max _{j<i} \operatorname{Pr}\left\{F_{i} \cap F_{j}\right\}
$$

Using this bound, the acceptable fraction is then approximated by

$$
A F=1-\sum_{i=1}^{m} \operatorname{Pr}\left\{F_{i}\right\}+\sum_{i=2}^{m} \max _{j<i} \operatorname{Pr}\left\{F_{i} \cap F_{j}\right\}
$$

Note that the last summation term of equation (10) is clearly dependent on the ordering of design requirements. Some researchers have proposed heuristic rules (Ditlevsen [56], Madsen et al [57]) while others have examined all $m$ ! permutations (Parkinson [58]). While the method in its most general form can accommodate nonlinear functional requirements and non-normal random variables, the stochastic tolerance chart allocation problem treated in this paper will be limited to the case where the output of manufacturing operations is adequately approximated by normal random variables.

Consider a functional requirement $g_{i}(\mathbf{x})=b_{i}+\sum_{j=1}^{n} a_{i j} x_{j} \geq 0$ and let $F_{i}$ represent the failure event associated with $g_{i}(\mathbf{x})<0$. If the $x_{j}$ variables are normal random variables (with parameters $\left(\mu_{j}, \sigma_{j}\right)$, a corresponding functional requirement $G_{i}(\mathbf{y})$ in standard normal 
space is readily obtained from $g_{i}(\mathbf{x})$ by way of a standard normal transformation and is given by:

$$
G_{i}(\mathbf{y})=b_{i}^{\prime}+\sum_{j=1}^{n} a_{i j}^{\prime} y_{j} \geq 0
$$

where

$$
y_{j}=\frac{x_{j}-\mu_{j}}{\sigma_{j}}
$$

Now define a safety margin $M_{i}$ by scaling $G_{i}(\mathbf{y})$ such that

$$
M_{i}(\mathbf{y})=\beta_{i}+\sum_{j=1}^{n} \alpha_{i j} y_{j} \geq 0
$$

where

$$
\alpha_{i j}=\frac{a_{i j}^{\prime}}{\sqrt{\sum_{j=1}^{n}\left(a_{i j}^{\prime}\right)^{2}}} \quad \text { and } \quad \beta_{i}=\frac{b_{i}^{\prime}}{\sqrt{\sum_{j=1}^{n}\left(a_{i j}^{\prime}\right)^{2}}}
$$

Note that $M_{i}=M_{i}(\mathbf{y})=0$ corresponds to the same failure surface $G_{i}(\mathbf{y})=0$ and that the minimum distance from the origin to the failure surface is equal to $\beta_{i}$. It is readily shown that the expected value of $M_{i}, \mathrm{E}\left(M_{i}\right)=\beta_{i}$ and the variance, $\operatorname{Var}\left(M_{i}\right)=1$. Since $M_{i}$ is also a normal random variable, it follows that

$$
\operatorname{Pr}\left\{F_{i}\right\}=\operatorname{Pr}\left\{g_{i}(\mathbf{x}) \leq 0\right\}=\operatorname{Pr}\left\{G_{i}(\mathbf{y}) \leq 0\right\}=\operatorname{Pr}\left\{M_{i} \leq 0\right\}=\Phi\left(\frac{0-\beta}{1}\right)=\Phi(-\beta)
$$

where $\Phi$ is the cumulative distribution function of the standard normal variable.

Using a similar approach, the probability associated with the intersection of failure events $F_{i}$ and $F_{j}$ is derived by considering corresponding safety margins $M_{i}$ and $M_{j}$ so that

$$
\operatorname{Pr}\left\{F_{i} \cap F_{j}\right\}=\operatorname{Pr}\left\{M_{i} \leq 0 \cap M_{j} \leq 0\right\}
$$

Since $M_{i}$ and $M_{j}$ are, in general, correlated normal random variables, the probability given by (11) can be written as

$$
\begin{aligned}
& \operatorname{Pr}\left\{M_{i} \leq 0 \cap M_{j} \leq 0\right\}= \\
& \int_{-\infty}^{-\beta_{i}-\beta_{j}} \int_{-\infty} \frac{1}{2 \pi \sqrt{1-\rho_{i j}^{2}}} \exp \left\{-\frac{1}{2\left(1-\rho_{i j}^{2}\right)}\left(x^{2}-2 \rho_{i j} x y+y^{2}\right)\right\} d x d y
\end{aligned}
$$


The numerical evaluation of the probability given by (12) is simplified by using the following expression (Owen [59])

$$
\begin{aligned}
& \operatorname{Pr}\left\{F_{i} \cap F_{j}\right\}=\operatorname{Pr}\left\{M_{i} \leq 0 \cap M_{j} \leq 0\right\}= \\
& \frac{1}{2 \pi} \int_{0}^{\rho_{i j}} \frac{1}{\left(1-z^{2}\right)^{1 / 2}} \exp \left\{-\frac{1}{2} \frac{\beta_{i}^{2}-2 \beta_{i} \beta_{j} z+\beta_{j}^{2}}{1-z^{2}}\right\} d z+\Phi\left(-\beta_{i}\right) \Phi\left(-\beta_{j}\right)
\end{aligned}
$$

where $\rho_{i j}=\sum_{k=1}^{n} \alpha_{i k} \alpha_{j k}, M_{i}=\beta_{i}+\sum_{k=1}^{n} \alpha_{i k} y_{k}>0$, and $M_{j}=\beta_{j}+\sum_{k=1}^{n} \alpha_{j k} y_{k}>0$.

The summation term, $\sum_{i=2}^{m} \max _{j<i} \operatorname{Pr}\left\{F_{i} \cap F_{j}\right\}$, in (10), is clearly dependent upon the ordering of the functional requirements $F_{i}, i=1, \ldots, m$ and three different ordering arrangements were considered for its evaluation. These approximations are based on the individual failure probabilities $\mathrm{P}\left(F_{i}\right)$ and the matrix of pairwise failure probabilities $\mathrm{P}\left(F_{i} \cap F_{j}\right)$. The first ordering sorts the constraints by placing the matrix row sums $\sum_{\substack{j=1 \\ j \neq i}}^{m} \mathrm{P}\left(F_{i} \cap F_{j}\right), i=1, \ldots, m$ in

ascending order. The second ordering sorts the constraints by placing the ratio of matrix row means to matrix row standard deviations in descending order. The third ordering sorts the constraints by placing the individual probabilities, $F_{i}, i=1, \ldots, m$ in descending order. Upon computing the summation term using each of the three ordering methods, the maximum value is retained for use.

The above described method was coded as a self contained program and implemented within the implicit enumeration algorithm to compute the probability of producing an acceptable part associated with a particular process combination.

Implicit Enumeration using FOSMM to Compute Yield: The implicit enumeration approach described earlier (see Figure 19) was used to determine the optimal tolerance allocation in the context of conventional tolerance control. However, in this case, the yield was computed using the FOSMM approach described in the previous section. Recall that it was necessary to estimate the yield via simulation in the STC approach.

The tolerance allocation problem corresponding to the part depicted in Figure 9 was solved in order to investigate the efficiency of the implicit enumeration algorithm. It was assumed that there are 4 process technologies available for each of the 9 required operations resulting in $4^{9}=262,144$ possible process combinations that need to be implicitly considered by this algorithm. The capability and cost of each process for the 9 operations are described in Table 5. FOSMM requires that each dimension be produced by an operation that follows a normal distribution. Therefore, it is assumed that the mean of the distribution corresponds to the 
nominal value of the dimension while the precision for each machine in Table 5 is related to the standard deviation of the distribution.

Due to the fathoming procedure described previously and the use of check nodes it was necessary to calculate the process yield for only 220 or $.08 \%$ of the possible process combinations. The minimum acceptable process yield was set at $99 \%$ for the solution of this part. Alternative optimal solutions were identified by the implicit enumeration procedure. Either combination (030101233) with an associated yield of $99.07 \%$ or combination (030102133) with an associated yield of $99.10 \%$ could be used to produce the part for a minimum cost of 51 .

In order to verify the accuracy of FOSMM the 10-operation part was also solved using a Monte Carlo simulation to determine the process yield. The results of this comparison are discussed in the next section.

Verification of FOSMM for Calculating Process Yields: The process yield can be determined via FOSMM as well as a Monte Carlo simulation. In order to demonstrate that FOSMM provides accurate yield calculations, the implicit enumeration algorithm was applied using both simulation and FOSMM. Recall that FOSMM is a conservative approximation of the true process yield. Therefore, it would be expected that process yields calculated via FOSMM would be slightly less than the yield obtained by simulation. It was determined that FOSMM computed a yield which was approximately .2\% lower that the yield calculated via simulation. In order to obtain this degree of agreement between the two methods it was necessary to simulate 100,000 parts. The simulation of parts within conventional tolerance control utilizes a vector of random deviates to model actual operations. The random deviates were drawn from a normal distribution with a standard deviation corresponding to the precision capability of the process. The mean of the distribution corresponds to the process plan set point.

As a result it is possible that FOSMM may obtain an optimal solution which has a higher associated cost. This would be the case if the optimal process combination obtained via simulation was slightly less than or equal to the minimal acceptable fraction of acceptable parts, while the optimal solution from FOSMM was slightly greater than the minimum acceptable fraction.

This in fact was the case when the 10-operation part was solved. The minimum fraction of acceptable parts is set at $99.0 \%$. Consider node (021101233) with a cost of 50. Both methods computed the yield of this combination. The simulation produced a feasible yield of $99.0 \%$ while FOSMM calculated the yield to be $98.7 \%$. This combination turned out to be the optimal solution for the simulation method. FOSMM's conservative approximation determined the combination to be infeasible and continued to search for an improvement to the incumbent solution. The optimal solution was determined to be combination (030101233) with an associated cost of 51. 
Conclusion: A new approach to the tolerance control problem in machining of discrete parts has been developed and presented. The approach makes effective use of measurements acquired at the end of operation $j$ to dynamically adjust the set point of operations $j+1$ to $n$. It is suitable for the production of low volume, high value added components. A comparative evaluation that compares this new approach [sequential tolerance control (STC)] and the conventional tolerance control approach shows that STC enables significantly better yields out of the precision of existing equipment. The frequency and location of break points for measurements and set point adjustment needs to be investigated for minimal intrusion on the process plan and objective evaluation of economic implications.

Variability in the manufactured dimensions of a part may be the result of both systematic variation such as tool wear and non-repeatable (random) variation in the manufacturing processes. A method was presented for using the techniques of STC to address the problem of tool-wear correction. In particular, based on the assumption of linearity, a slope approximation method was developed to predict and compensate for tool wear in the framework of STC. Simulation results demonstrate the viability of the methodology in reducing the number of defective parts.

An implicit enumeration approach to tolerance allocation using STC was also presented. The monotone structure of the probability of producing a good part as a function of process precision and cost was exploited in fathoming the search tree. An efficient algorithm was developed to determine process combinations that minimize manufacturing cost while maintaining an acceptable proportion of good parts. In addition it was demonstrated that the use of STC would result in substantial savings in manufacturing cost as compared to conventional tolerance control. Issues regarding more efficient fathoming of the process combination tree are currently being investigated.

Finally, An implicit enumeration approach to tolerance allocation using conventional tolerance control was developed in which process yield was calculated using FOSMM. In addition, the accuracy of FOSMM was verified through a comparison to results obtained via simulation.

Acknowledgment: This research is based on work supported by National Science Foundation Grant No. DMI-9622620.

\section{References}

[1] Gadzala, J.L., 1959, Dimensional Control in Precision Manufacturing, McGraw Hill, New York.

[2] Eary, D.F., Johnson, G.E., 1962, Process Engineering for Manufacturing, Prentice Hall, Englewood Cliffs, NJ.

[3] Wade, O.R., 1967, Tolerance Control in Design and Manufacturing, Industrial Press, Inc., New York. 
[4] Patel, A.M., Computer-Aided Assignment of Manufacturing Tolerances, ACM 089791-020-6/80/0600/0129 (reprint).

[5] Wade, O.R., 1983, Tolerance Control, Tool and Manufacturing Engineers Handbook, Vol. 1, Machining, Society of Manufacturing Engineers, Dearborn, Michigan, pp. 2-1 2-60.

[6] Ahluwalia, R.S., Karolin, A.V., 1986, CATC -- A Computer Aided Tolerance Control System. Journal of Manufacturing Systems 3, 153-160.

[7] Fainguelernt, D. Weill, R. Bourdet, P., 1986, Computer Aided Tolerancing and Dimensioning in Process Planning, ANNALS of the C.I.R.P. 35, 381-386.

[8] Dong, Z., Soom, A., 1986. Automatic Tolerance Analysis from a CAD Database. ASME Technical Paper 86-DET-36, pp. 1-8.

[9] Xiaoqing, T. Davies, B.J., 1988. Computer Aided Dimensional Planning. International Journal of Production Research 26, 283-297.

[10] Irani, S.A., Mittal, R.O., Lehtihet, E.A., 1989, Tolerance Chart Optimization, International Journal of Production Research 27, 1531-1552.

[11] Mittal, R.O., Irani, S.A., Lehtihet, E.A., 1990. Tolerance Control in the Machining of Discrete Components. Journal of Manufacturing Systems 9(3), 233-246.

[12] Dong, Z., Hu, W. 1991. Optimal Process Sequence Identification and Optimal Process Tolerance Assignment in Computer-Aided Process Planning. Computers in Industry 17, 19-32.

[13] Ngoi, B.K.A., 1992, Applying Linear Programming to Tolerance Chart Balancing, International Journal of Advanced Manufacturing Technology 7, 187-192.

[14] Abdou, G., Cheng, E. 1993, TVCAPP, Tolerance Verification in Computer-Aided Process Planning, International Journal of Production Research 31(2), 393-411.

[15] Ngoi, B.K.A., Ong, C.T., 1993, A Complete Tolerance Charting System, International Journal of Production Research 31(2), 453-469.

[16] Ngoi, B.K.A., Fang, S.L., 1994, Computer-Aided Tolerance Charting, International Journal of Production Research 32(8), 1939-1954.

[17] Fraticelli, B.P, Lehtihet, E.L, Cavalier, T.M., 1997, Sequential Tolerance Control in Discrete Parts Manufacturing, International Journal of Production Research Vol. 35, 
No. 5, 1305-1319.

[18] Duret, D. 1988. Cotation et Methods de Controle en Fabrication Mecanique, Chapters 4 and 8 (Evolutive Tolerancing), Editions Augustin, Paris.

[19] Hunter, W.G., Kartha, C.P., 1977, Determining the Most Profitable Target Value for a Production Process, Journal of Quality Technology 9, 176-181.

[20] Bjorke, O., 1978, Computer-Aided Tolerancing, Tapir Publishers, Norway.

[21] Arcelus, F.J., Banerjee, P.K., 1985. Selection of the Most Economical Production Plan in a Tool-Wear Process. Technometrics, 27(4), 433-436.

[22] Pugh, G.A., 1988, An Algorithm for Economically Setting a Uniformly-Shifting Process, Computers and Industrial Engineering 14, 237-240.

[23] Quesenberry, C.P., 1988. An SPC Approach to Compensating a Tool-Wear Process. Journal of Quality Technology, 20(4), 220-229.

[24] Drezner, Z, Wesolowsky, G.O., 1989. Control Limits for a Drifting Process with Quadratic Loss, International Journal of Production Research 27, 13-20.

[25] Jensen, K.L., Vardeman, S.B., 1993, Optimal Adjustment in the Presence of Deterministic Process Drift and Random Adjustment Error, Technometrics 35, 376-381.

[26] Rattanasoontorn, M., Chandra, M.J., 1995. The Economic Design of X Control Chart for Production Processes Subject to Linear Drift and a Single Assignable Cause. Working Paper \#95-113. Department of Industrial and Manufacturing Engineering, The Pennsylvania State University.

[27] Fraticelli, B.P, Lehtihet, E.L, Cavalier, T.M., 1997, Tool-Wear Effect Compensation Under Sequential Tolerance Control, forthcoming in International Journal of Production Research.

[28] Mansour, E.N., 1963, The Application of Probability to Tolerances Used in Engineering Design, Proc. Int. Mech. Engr., Vol. 178, Pt. 1, No. 1, $29-51$.

[29] Moy, William A., 1964, Assignment of Tolerances by Dynamic Programming, Machine Design, May 21, 215-218.

[30] Gladman, C.A., 1966, Techniques for Applying Probability to the Tolerancing of Machined Dimensions, Technical Paper No. 22, National Standards Laboratory, CSIRO, Sydney, Australia.

[31] Peters, J., 1970, Tolerancing the Components of an Assembly for Minimum Cost, 
ASME Publication Paper No. 70, Prod. 9.

[32] Bennett, G., Gupta, L.C., 1970a, Least-Cost Tolerances-1, International Journal of Production Research 8(1), 65-73.

[33] Bennett, G., Gupta, L.C., 1970b, Least-Cost Tolerances-2, International Journal of Production Research 8(2), 169-181.

[34] Speckhart, F.H., 1972, Calculation of Tolerance Based on Minimum Cost Approach, Transactions of the ASME Journal of Engineering for Industry, May, 447-453.

[35] Spotts, M.F., 1972, Allocation of Tolerances to Minimize Cost of Assembly, ASME Paper No. 72-WA1DE-6: 1-3.

[36] Spotts, M.F., 1975, Probability Theory for Assemblies with Part Errors Concentrated Near End of Tolerance Limit, ASME Paper No. 75-DE-1.

[37] Wilde, D., Prentice, E., 1975, Minimum Exponential Cost Allocation of Sure-Fit Tolerances, Transactions of the ASME Journal of Engineering for Industry, November, 1395-1398.

[38] Wilde, D., 1975, Simplifying Discrete Tolerance Assignment, ASME Paper No. 75DET-106: 1-7.

[39] Ostwald, P.F., Huang, J., 1977, A Method for Optimal Tolerance Selection, Transactions of the ASME Journal of Engineering for Industry, August, 558-565.

[40] Michael, W., Siddall, J.N., 1981, The Optimization Problem with Optimal Tolerance Assignment and Full Acceptance, ASME Journal of Mechanical Design 103, October, 842-848.

[41] Michael, W., Siddall, J.N., 1982, The Optimal Tolerance Assignment with Less Than Full Acceptance, ASME Journal of Mechanical Design 104, October, 855-860.

[42] Monte, M.E., Datseris, P., 1982, Optimal Tolerance Selection for Minimum Manufacturing Cost and other Design Criterion, ASME Paper No. 82-DET-35.

[43] Parkinson, D.B., 1982, The Application of Reliability Methods to Tolerancing, ASME Journal of Mechanical Design 104, July, 612-618.

[44] Parkinson, D.B., 1984, Tolerancing of Component Dimensions in CAD, Computer Aided Design 16(1):25-32.

[45] Parkinson, D.B., 1985, Assessment and Optimization of Dimensional Tolerances, Computer Aided Design 17(4):191-199. 
[46] Balling, R.J., Free, J.C., Parkinson, A.R., 1986, Consideration of Worst Case Manufacturing Tolerances in Design Optimization, ASME Journal of Mechanisms, Transmissions, and Automation in Design, Vol.108, 438-441.

[47] Lee, W.J., Woo, T.C., 1989, Optimum Selection of Discrete Tolerances, ASME Journal of Mechanisms, Transmissions, and Automation in Design, Vol.111, 243-251.

[48] Lee, W.J., Woo, T.C., Chou, S.Y., 1993, Tolerance Synthesis for Non-Linear Systems Based on Nonlinear Programming, IIE Transactions, Vol. 25, No 1, 51-61.

[49] Balakrishan, N. 1993, A Multiple Choice Knapsack Model for Tolerance Allocation in Mechanical Assemblies, IIE Transactions, Vol. 25, No. 4, 13-14.

[50] Varghese, P. Braswell, R.N., Wang, B., Zhang, C., 1996, Statistical Tolerance Analysis using FRPDF and Numerical Convolution, Computer Aided Design, Vol. 28, No. 9, 723-732.

[51] Wheeler, D.L., Cavalier, T.M., Lehtihet, E.L., 1997, An Implicit Enumeration Approach to Tolerance Allocation in Sequential Tolerance Control, forthcoming in IIE Transactions.

[52] Wheeler, D.L., Cavalier, T.M., Lehtihet, E.L., 1998, An Implicit Enumeration Approach to Probabilistic Tolerance Allocation under Conventional Tolerance Control, submitted to International Journal of Production Research.

[53] Balling, R.J., Free, J.C., Parkinson, A.R., 1986, Consideration of Worst Case Manufacturing Tolerances in Design Optimization, ASME Journal of Mechanisms, Transmissions, and Automation in Design, Vol.108, 438-441.

[54] Pandya, G., A Computer Assisted Tolerance Allocation System for Dominant Datum Systems in Product Design, M.S. Thesis, The Pennsylvania State University, University Park, May 1998.

[55] Melchers, R.E., 1987, Structural Reliability, Analysis, and Prediction, Ellis Horwood (Wiley), N.Y.

[56] Ditlevsen, O., 1979, Narrow Reliability Bounds for Structural Systems, Journal of Struct. Mech., Vol. 7, No. 4, 453-472.

[57] Madsen, H.O., Krenk, S., Lind, N.C., 1986, Methods of Structural Safety, Prentice Hall, Englewood Cliff, N.J.

[58] Parkinson, D.B., 1988, Reliability Bounds for Dependent Failures, IEEE Transactions on Reliability, Vol. 37, No. 1. 
[59] Owen, D.B., 1956, Tables for Computing Bivariate Normal Probabilities, Annals of Mathematical Statistics, Vol. 27, 1075-1090. 